\title{
Use of transcranial magnetic stimulation in the treatment of selected movement disorders
}

This article was published in the following Dove Press journal:

Degenerative Neurological and Neuromuscular Disease

4 December 2014

Number of times this article has been viewed

\author{
Katlyn E Brown' \\ Jason L Neva ${ }^{2}$ \\ Noah MH Ledwell' \\ Lara A Boyd ${ }^{1,2}$ \\ 'Graduate Program in Rehabilitation \\ Science, ${ }^{2}$ Department of Physical \\ Therapy, Faculty of Medicine, \\ University of British Columbia, \\ Vancouver, BC, Canada
}

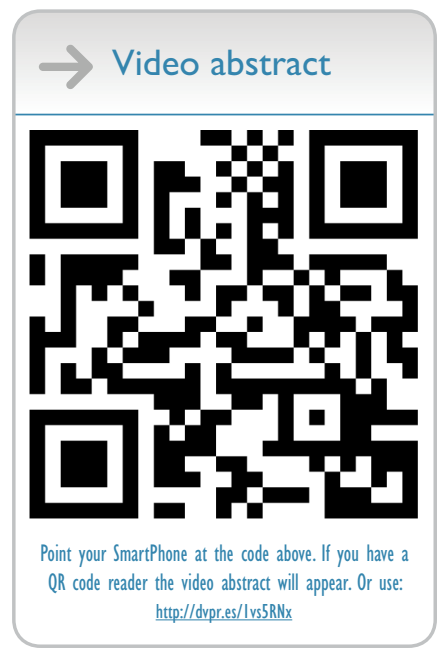

Correspondence: Lara A Boyd Department of Physical Therapy, Faculty of Medicine, University of British Columbia, 212-2I77 Wesbrook Mall,

Vancouver, BC V6T IZ3, Canada

Tel +I 6048273369

Fax +l 6048221870

Email lara.boyd@ubc.ca

\begin{abstract}
Transcranial magnetic stimulation (TMS) is a valuable technique for assessing the underlying neurophysiology associated with various neuropathologies, and is a unique tool for establishing potential neural mechanisms responsible for disease progression. Recently, repetitive TMS (rTMS) has been advanced as a potential therapeutic technique to treat selected neurologic disorders. In healthy individuals, rTMS can induce changes in cortical excitability. Therefore, targeting specific cortical areas affected by movement disorders theoretically may alter symptomology. This review discusses the evidence for the efficacy of rTMS in Parkinson's disease, Huntington's disease, amyotrophic lateral sclerosis, and multiple sclerosis. It is hoped that gaining a more thorough understanding of the timing and parameters of rTMS in individuals with neurodegenerative disorders may advance both clinical care and research into the most effective uses of this technology.
\end{abstract}

Keywords: repetitive transcranial magnetic stimulation, selected movement disorders, treatment

\section{Introduction}

Transcranial magnetic stimulation (TMS) is a noninvasive technique that quantifies levels of cortical and spinal excitability. TMS, in its simplest form, involves a single stimulus delivered over the scalp above the target cortical area to be probed. TMS relies on the principle of electromagnetic induction to excite cortical neurons. A figure-of- 8 coil contains two wires. When triggered, current flows along these wires in opposite directions. The magnetic field created by the coil where the wires cross traverses the scalp and the change in magnetic field generates electrical activity within the surface of the cortex. The stimulation results in altered membrane potentials, which lead to action potentials, inhibitory postsynaptic potentials, or excitatory postsynaptic potentials (Figure 1). More specifically, intracortical interneurons activated by TMS are likely axons of pyramidal neurons in layers II and III of the primary motor cortex (M1). These neurons in layers II and III then synapse onto pyramidal corticospinal output neurons in layer V of the cortex. Generally, these intracortical interneurons are thought to be activated with the TMS coil orientation 45 degrees to the mid-sagittal line held over the primary motor cortex, which generates a posterior to anterior current (depending on the current generated within the TMS coil) and produces what are described as multiple I-waves (I denotes indirect). However, if the TMS coil orientation is 90 degrees (perpendicular) to the mid-sagittal line held over the primary motor cortex, pyramidal corticospinal output neurons in layer $\mathrm{V}$ are activated more directly and produce what are described as D-waves 
A

Electrical field produced in cortex

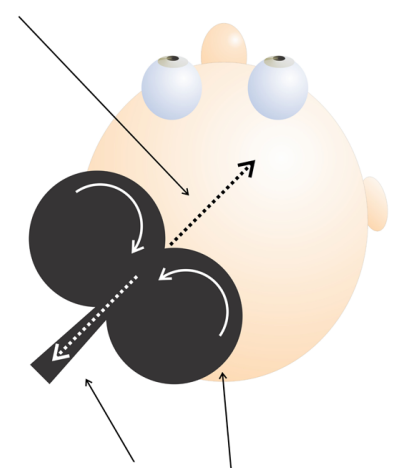

Electrical field produced in coil
B

Electrical field in coil Magnetic field

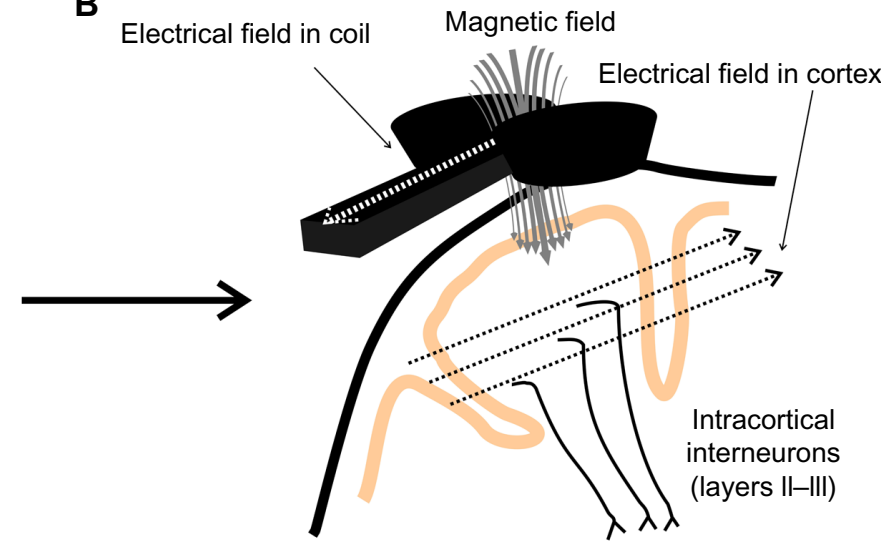

D

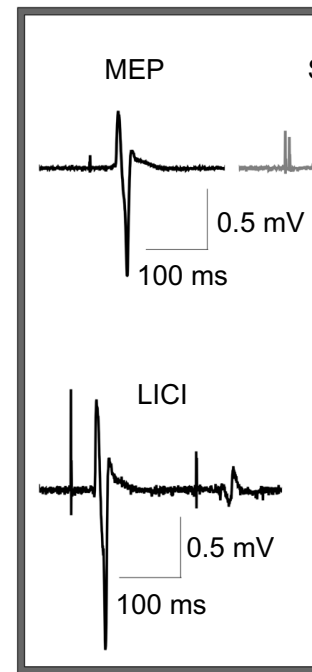

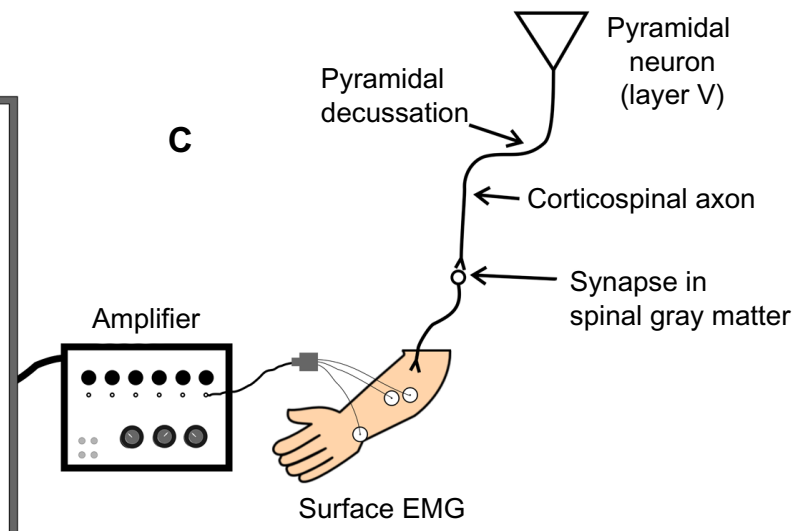

Motor evoked potentials

Figure I Schematic of transcranial magnetic stimulation (TMS).

Notes: (A) A TMS coil placed over the scalp produces an electric field in the TMS coil, as well as in the opposite direction in the cortex. (B) Electric field produced by the coil induces a magnetic field traversing the skull and dura, producing electrical activity in the cortex in the opposite direction, which activates intracortical interneurons synapsing on pyramidal neurons and thus activates the corticospinal tract. The descending corticospinal tract then synapses on to a motor neuron in the spinal gray matter, which then carries the signal to the muscle. (C) The electromyographical (EMG) response resulting from TMS-induced activation of the corticospinal tract is captured via electrodes recording from the target muscle. (D) Amplified EMG is projected onto a computer screen for quantification of motor evoked potential (MEP) amplitude and latency. The computer screen displays examples of TMS-induced MEP, short-interval intracortical inhibition (SICl), intracortical facilitation (ICF), long-interval intracortical inhibition ( $\mathrm{LICl})$, and cortical silent period (CSP).

(D denoting direct). Increasing the intensity of a single TMS pulse could potentially activate a greater area and number of neurons, and also may penetrate deeper into the layers of the cortex. Given that the dominant pattern of activity involves activation of I-waves, TMS-evoked responses are sensitive to changes in both interneuronal populations and corticospinal excitability (for review, see Di Lazzaro and Ziemann ${ }^{1}$ ).

TMS can theoretically be used on any cortical region; however, M1 is an optimal candidate as electromyography provides a quantifiable outcome measure. In order to establish baseline levels of M1 excitability, or inhibition, TMS can be used in various ways (Tables $1-3$ ), as outlined below.
Baseline measures can be compared between healthy controls and individuals with various pathologies to understand the effect on patterns of cortical excitation and inhibition. Additionally, these techniques can be applied prior to, and following, an intervention designed to induce neuroplastic change or explore potential therapeutic benefits.

\section{TMS techniques}

Single-pulse and paired-pulse TMS techniques are primarily used to quantify levels of cortical excitability and inhibition. When TMS is used in a repetitive manner, changes in cortical excitability lasting longer than the duration of stimulation can 


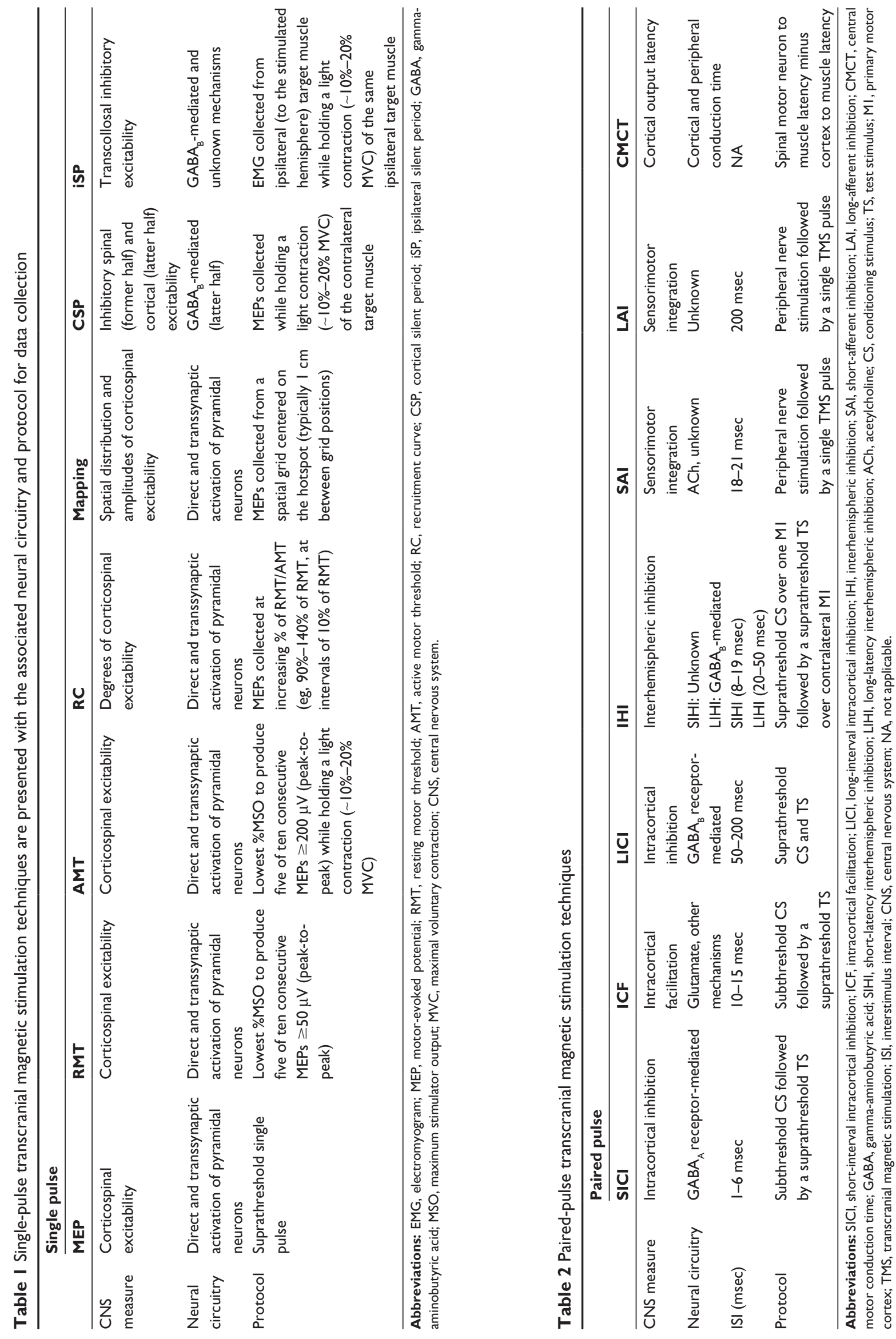




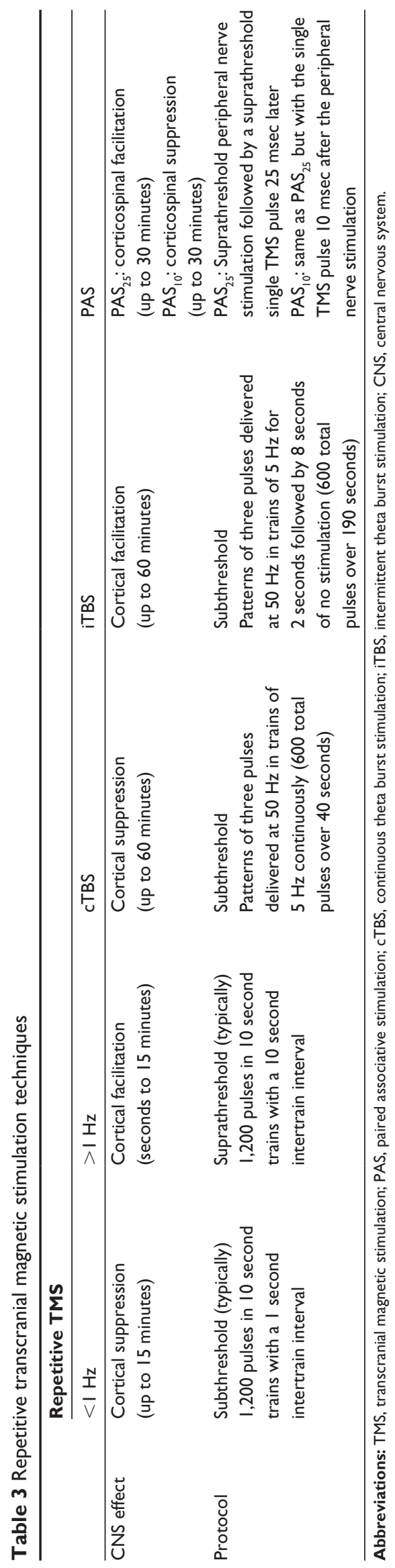

occur. This method has been implicated as a potential therapeutic resource or treatment for many neurological disorders. Additionally, single-pulse and paired-pulse TMS techniques can be used in conjunction with repetitive TMS (rTMS)based interventions to quantify neurophysiological changes resulting from the intervention that may not be apparent with behavioral outcome measures. The neurophysiological mechanisms thought to underlie the various TMS techniques described can be found in Tables 1-3.

\section{Single-pulse TMS \\ Motor thresholds (MTs)}

Single-pulse TMS over M1 produces muscle responses in a well-defined waveform referred to as a motor-evoked potential (MEP). In order to standardize responses between individuals, a value of MT is typically determined. Resting MT is defined as the percent of stimulator output that is required to produce an MEP with a peak-to-peak amplitude of $50 \mu \mathrm{V}$ on five of ten trials, while the individual is at rest. ${ }^{2}$ Similarly, active MT is defined as the percent of stimulator output that is required to produce an MEP with a peak-to-peak amplitude of $200 \mu \mathrm{V}$ on five of ten trials, while the individual maintains a background contraction. ${ }^{3}$ Threshold values are often used to determine the intensity of stimulator output in many of the TMS techniques used to assess and treat various movement disorders.

\section{Recruitment curves}

Recruitment curves utilize single-pulse TMS over a range of intensities to measure the increase in excitability within the corticospinal tract in response to increased stimulus intensity, as indexed by MEP amplitude. ${ }^{4}$ The slope of the curve produced by increasing stimulator intensity is quantified as a representation of the ability of the excitability of the primary M1 to be upregulated, and the strength of the corticospinal connections. ${ }^{5}$ Recruitment curves can be measured while the participant is at rest, or during a sustained contraction. Resting recruitment curves activate lower threshold neurons, while active recruitment curves utilize the voluntary contraction to activate higher threshold neurons, thus stimulating unique neuronal pools, which may have different functional significance. $^{6}$

\section{MI cortical mapping}

Single-pulse TMS can also be utilized to probe the excitability of M1 in terms of quantifying the distribution and amplitudes of MEP in the target muscles. To map a muscle representation in M1, the motor representational "hotspot" 
is found. Next, the location of stimulation is moved out in each direction (lateral, medial, anterior, posterior) by $1 \mathrm{~cm}$ increments until MEPs from the target muscle are no longer elicited. Mapping the M1 representation of particular muscles is used to understand normal and pathological cortex, as well as to map change in muscle representation over time or following an intervention. ${ }^{7-16}$

\section{Silent period}

Active electromyographic responses to single-pulse TMS include a cortical silent period (CSP), which presents as a prolonged reduction in electromyographic activity following the MEP. CSPs have been shown to be dependent on recovery from inhibition at the level of M1. ${ }^{17-19}$ Therefore, single-pulse TMS can be indicative not only of motor cortical excitability, or increases in corticospinal tract excitability in response to increasing stimulator output, but also inhibitory circuit activity within M1.

Transcallosal inhibition, important in interhemispheric communication, can be quantified via an ipsilateral silent period derived from TMS protocols. Specifically, during a sustained unilateral muscle contraction, a single TMS pulse over the ipsilateral $\mathrm{M} 1$ is delivered to evoke a silent period in the background activity generated by the ipsilateral muscle, known as the ipsilateral silent period.

\section{Paired-pulse TMS}

\section{Intracortical inhibition and facilitation}

The excitation of M1 pyramidal neurons that ultimately translates into movement does not occur in isolation. Inhibitory interneurons within M1 influence this output, and can also be quantified using TMS. Paradigms, specifically short-interval intracortical inhibition (SICI) and long-interval intracortical inhibition (LICI), quantify inhibitory circuitry. SICI occurs when two TMS pulses (a subthreshold conditioning stimulus followed by a suprathreshold test stimulus) are administered over M1 with an interstimulus interval (ISI) of 1-6 msec and result in a decreased MEP amplitude when compared with that elicited from a single TMS pulse alone. ${ }^{20}$

A period of intracortical facilitation (ICF), $10-15 \mathrm{msec}$ after the stimulation, can quantify the facilitatory circuits in M1. The protocol for extracting this is identical to that seen with SICI (subthreshold conditioning stimulus and suprathreshold test stimulus), with only the ISI differing.

Following a window in which ICF is present, LICI occurs at an ISI of 50-200 msec. ${ }^{21,22}$ Unlike SICI, LICI is evoked with two suprathreshold pulses. Both SICI and LICI are important for neuroplasticity, whether it is related to behavioral-dependent or stimulation-dependent interventions. Assessing these inhibitory circuits is an important component of understanding how neuroplastic change contributes to functional improvement and is instrumented in M1.

\section{Central motor conduction time}

In order to quantify central motor conduction time (CMCT), TMS is used in conjunction with peripheral nerve stimulation. Peripheral nerve stimulation produces an F-wave, which is indicative of time to travel in an antidromic direction from the peripheral nerve to the spinal cord, and back to the muscle. The M-wave represents orthodromic conduction arising from peripheral nerve stimulation to directly stimulate the corresponding muscle. When added together, the F-wave and $\mathrm{M}$-wave latencies provide a measure of the peripheral conduction time. MEP latency is indicative of both central and peripheral conduction times. Therefore, subtracting the F-wave and M-wave latency in one direction from the overall MEP latency offers a measure of central nervous system conduction time. ${ }^{23}$

\section{Short-afferent and long-afferent inhibition}

Short-afferent inhibition and long-afferent inhibition use TMS, in conjunction with peripheral nerve stimulation, to examine the integration of sensory information into M1. Specifically, an electrical stimulation is delivered at the contralateral median nerve prior to a TMS pulse delivered over M1 while the participant is at rest. Short-afferent inhibition applies this technique with an ISI of $20 \mathrm{msec}$ and long-afferent inhibition utilizes an ISI of $200 \mathrm{msec}^{17,24-28} \mathrm{The}$ short ISI associated with short-afferent inhibition provides only enough time for activation of the primary somatosensory cortex and secondary somatosensory cortex, whereas the longer ISI associated with long-afferent inhibition is long enough to ensure activation of primary somatosensory cortex, bilateral secondary somatosensory cortex, and contralateral posterior parietal cortex. ${ }^{17}$ While the mechanisms underlying both short-afferent inhibition and long-afferent inhibition have yet to be elucidated, they provide information on the impact of peripheral nerve stimulation on M1 excitability, which is an important component to consider when studying sensorimotor integration in regards to neuroplasticity.

\section{Repetitive stimulation}

In addition to single-pulse and paired-pulse techniques, TMS can be applied in a repetitive manner. rTMS can be applied in specific patterns to modulate cortical excitability; the effects 
of rTMS may last for periods of time that exceed that of stimulus application. As a result, rTMS can be used to index neuroplasticity or as a potential treatment.

\section{Paired associative stimulation}

Paired associative stimulation (PAS) exploits the principles of associative plasticity to engage long-term potentiation-like mechanisms and produce lasting increased excitability within M1. PAS pairs the afferent information from peripheral nerve stimulation with a synchronously delivered TMS pulse over M1. ${ }^{29}$ Specifically, PAS involves a peripheral nerve stimulation (usually to the median nerve, activating a muscle like the abductor pollicis brevis) which is then followed by a single pulse of TMS over the abductor pollicis brevis muscle representation contralateral to the peripheral nerve stimulation 20-25 msec later. The PAS protocol usually involves 180 total pairs of these stimuli, with an ISI of 4-10 msec. MEPs are increased following the PAS protocol in the specific muscle targeted (usually abductor pollicis brevis) for a period of time (about 30 minutes).

\section{Repetitive TMS}

TMS, when applied in specific patterns, can be used to excite or inhibit a local cortical region for a short duration. rTMS can be applied at low frequencies of under $1 \mathrm{~Hz}$, which suppress excitability in the targeted area, or at high frequencies over $1 \mathrm{~Hz}$, which transiently excite the targeted area for approximately 15 minutes. ${ }^{30}$ Similarly, theta burst stimulation (TBS) uses a theta stimulation pattern to inhibit or facilitate cortical excitability if the TBS is applied continuously (inhibitory cTBS), or intermittently (facilitatory iTBS), respectively. ${ }^{31}$ The effects of cTBS and iTBS can last up to 60 minutes post stimulation. ${ }^{31,32}$ Importantly, the specific effects of cTBS and iTBS show substantial interindividual variability, which likely depends upon which interneuron populations are activated by the TMS pulse. ${ }^{33}$ rTMS protocols, like TBS, have been shown to modulate cortical excitability, and at times behavior, when applied over motor-related areas, such as M1, contralateral $\mathrm{M} 1,{ }^{34,35}$ the supplementary motor area (SMA), ${ }^{36}$ the dorsal premotor cortex (PMd), ${ }^{37}$ the primary somatosensory cortex, area $5,{ }^{38}$ as well as non-motor areas such as the cerebellum ${ }^{39}$ and the dorsal lateral prefrontal cortex (DLPFC). ${ }^{40}$ Furthermore, rTMS is currently a treatment for drug-resistant major depressive disorder. ${ }^{41}$ These methods for changing cortical excitability are thought to mimic early long-term potentiation or long-term depression-type mechanisms, and are dependent upon N-methyl D-aspartate receptors. ${ }^{42}$ Due to this similarity to previously documented long-term potentiation, researchers have developed protocols to test whether the application of this stimulation alone, or in conjunction with other therapy, can further rehabilitation or treatment strategies.

The successful use of TMS in a variety of neuropathologies such as depression, Alzheimer's disease, schizophrenia, and stroke, supports the potential role of TMS as an intervention in neurologic disorders/diseases. The evidence for TMS both as an intervention, as well as an assessment tool, is outlined in the present paper for Parkinson's disease (PD), Huntington's disease (HD), multiple sclerosis (MS), and amyotrophic lateral sclerosis (ALS). Currently, while these diseases have a variety of treatment options to slow their progression, an optimal treatment solution that reverses or halts their course has not been found. It is possible that TMS could be employed in conjunction with current treatments to improve clinical interventions as well as to advance understanding of specific disease processes.

\section{Parkinson's disease}

$\mathrm{PD}$ is a neurodegenerative disorder characterized by tremor, rigidity, bradykinesia/akinesia, and postural instability. ${ }^{43}$ The specific neural degeneration involves the substantia nigra (pars compacta) dopaminergic-producing neurons within the subcortical basal ganglia. ${ }^{43}$ Although PD is associated with degeneration of striatal subcortical structures, many neuroimaging studies have identified common patterns of cortical activation abnormalities that may be associated with functional impairments. ${ }^{44} 46$ Use of multiple TMS techniques has provided insight into the underlying cortical mechanisms involved in PD. This section briefly introduces assessment of the underlying cortical mechanisms of PD and primarily focuses on rTMS-induced modulation of cortical excitability as a treatment for PD-related abnormalities in cortical activity and motor behavior.

\section{Assessment}

Several TMS studies have investigated the potential neural mechanisms contributing to the symptomology of PD, and show both common and conflicting findings. Measures of cortical excitability abnormalities in PD are summarized in Tables 4-6. In general, individuals with PD show paradoxical hyperexcitability and hypoexcitability along with impaired plasticity as measured by TMS. For example, MTs and CMCT are generally normal ${ }^{47-49}$ while motor maps are larger and more excitable. ${ }^{50}$ Additionally, generally CSP duration is shorter, ${ }^{47,51,52} \mathrm{SICI}^{49,53-55}$ and $\mathrm{ICF}^{56-58}$ are reduced, and LICI is enhanced. ${ }^{53,55,59}$ Conflicting findings could be due to disease progression, duration, presence of levodopa-induced 
Table 4 Summary of pathology-related changes in single-pulse transcranial magnetic stimulation-based measures of neurophysiology

\begin{tabular}{|c|c|c|c|c|c|c|c|}
\hline & \multicolumn{7}{|l|}{ Single-pulse TMS } \\
\hline & MEP & RMT & AMT & $\mathbf{R C}$ & Mapping & CSP & iSP \\
\hline PD & & $\begin{array}{l}\mathrm{H}_{0}^{47} \\
\downarrow^{47}\end{array}$ & $\begin{array}{l}\mathrm{H}_{0}^{47} \\
\uparrow_{47}\end{array}$ & & $\uparrow$ area $^{50}$ & $\downarrow^{47,51,52}$ & \\
\hline HD & $\begin{array}{l}\mathrm{H}_{0}^{119} \\
\downarrow^{211,212}\end{array}$ & $\begin{array}{l}\uparrow_{123} \\
\mathrm{H}_{0}^{89}\end{array}$ & $\mathrm{H}_{0}^{89}$ & $\downarrow$ slope $e^{123}$ & & $\begin{array}{l}\uparrow 120,212-214 \\
\downarrow 121,215 \\
\mathrm{H}_{0}^{89,122}\end{array}$ & \\
\hline ALS & $\begin{array}{l}\uparrow_{128,152,156} \\
\mathrm{H}_{0} \text { latency }{ }^{129,130,150} \\
\uparrow_{\text {latency }}^{129-131,150,153,154,165,216}\end{array}$ & $\begin{array}{l}\uparrow \mid 29,130,132,150,153,154,165,216-219 \\
\downarrow 130,133,134,218,220\end{array}$ & & $\downarrow^{161}$ & $\downarrow$ area $^{221}$ & $\begin{array}{l}\downarrow 128,130,132,136,156,159,162, \\
178,179,222,223 \\
\uparrow 161\end{array}$ & $\begin{array}{l}\uparrow 137,161,226 \\
\text { Absent }^{137,161,214,226}\end{array}$ \\
\hline MS & $\begin{array}{l}\downarrow^{161} \\
\uparrow \text { latency }^{13,172,174,185} \\
\mathrm{H}_{0}^{172} \\
\downarrow \text { amplitude }{ }^{13,172,174,181}\end{array}$ & $\begin{array}{l}\mathrm{H}_{0}^{135,161} \\
\mathrm{H}_{0}^{180}\end{array}$ & $\begin{array}{l}\uparrow \mathrm{rel}^{|8|} \\
\mathrm{H}_{0} \mathrm{rem}^{181}\end{array}$ & & & $\begin{array}{l}\mathrm{H}_{0}^{135} \\
\uparrow_{174} \\
\uparrow \mathrm{rem}^{181} \\
\downarrow \mathrm{rel}^{181}\end{array}$ & $\downarrow^{135}$ \\
\hline
\end{tabular}

Notes: Mapping refers to the spatial distribution of MEPs over the surface of the scalp.

Abbreviations: TMS, transcranial magnetic stimulation; MEP, motor-evoked potential; RMT, resting motor threshold; AMT, active motor threshold; RC, recruitment curve; Mapping, the spatial distribution of MEPs over the surface of the scalp; CSP, cortical silent period; iSP, ipsilateral silent period; $\mathrm{H}_{0}$, null result; $\uparrow$, increase; $\downarrow$, decrease; rel, relapsing; rem, remitting; ALS, amyotrophic lateral sclerosis; PD, Parkinson's disease; MS, multiple sclerosis; HD, Huntington's disease.

dyskinesia (LID), medication cycles, asymmetry of symptoms, ${ }^{51}$ and TMS methods. Many of the TMS-based neurophysiological measures do not correlate with symptoms and motor behavior. However, there are data linking alterations in motor function (rigidity, ${ }^{47}$ bradykinesia, ${ }^{48}$ Unified Parkinson's Disease Rating Scale [UPDRS] scores from the affected side ${ }^{60}$ ) with TMS-based measures of motor cortical excitability.

\section{Treatment}

There have been an increasing number of studies employing rTMS to treat the symptoms of PD (including abnormal cortical excitability in PD). These show wide variability (and at times conflicting) results regarding the ability of rTMS to modulate cortical excitability in individuals with PD, possibly due to utilizing varying frequencies, durations, intensities, and stimulation targets. In general, it seems that, depending on the cortical location of application, early-stage or late-stage PD, and those that display specific motor and psychological symptoms, selection of high frequency or low frequency rTMS can produce beneficial after-effects in cortical excitability and the associated function. This section summarizes the current state of the literature regarding the use of rTMS to treat the symptoms of PD and provides information regarding the associated cortical activity modulations. The most common cortical locations of rTMS for the treatment of PD are M1, DLPFC, SMA, PMd, and the cerebellum, which are discussed below.

\section{rTMS over MI}

The basal ganglia-thalamo-cortical drive to M1 is a potential circuit involved in the motor deficits associated with $\mathrm{PD}$, making M1 a plausible target for rTMS.${ }^{61}$ Studies have shown abnormal (hyperexcitability) within M1 in those with PD. ${ }^{44,45,62,63}$ Previous studies have shown that $10 \mathrm{~Hz}$ rTMS over M1 can stimulate release of dopamine in the

Table 5 Summary of pathology-related changes in paired-pulse transcranial magnetic stimulation-based measures of neurophysiology

\begin{tabular}{|c|c|c|c|c|c|c|c|}
\hline & \multicolumn{7}{|l|}{ Paired-pulse TMS } \\
\hline & SICl & ICF & LICl & IHI & SAI & LAI & СMCT \\
\hline \multirow[t]{2}{*}{ PD } & $\downarrow$ rest ${ }^{49,53-55}$ & $\downarrow^{56-58}$ & $\uparrow 53,5,59$ & $\mathrm{H}_{0}^{188}$ & $\mathrm{H}_{0}^{28}$ & $\downarrow^{28}$ & $\mathrm{H}_{0}^{49}$ \\
\hline & $\mathrm{H}_{0}$ active $^{59,186}$ & & $\downarrow^{187}$ & $\downarrow^{188}$ & $\downarrow 28$ & & \\
\hline \multirow[t]{2}{*}{ HD } & $\downarrow 89,211$ & $\uparrow 189$ & & & $\downarrow^{123}$ & & $\mathrm{H}_{0}^{89,119,190}$ \\
\hline & $\mathrm{H}_{0}^{189}$ & $\downarrow 89$ & & & & & \\
\hline \multirow[t]{2}{*}{ ALS } & $\downarrow \mid 128,136,138,139,152,156,199-193,224$ & $\uparrow 152$ & & & & & $\uparrow 130,152$ \\
\hline & & & & & & & $\mathrm{H}_{0}{ }^{129-131,150,153,154,165,216}$ \\
\hline \multirow[t]{3}{*}{ MS } & $\downarrow \downarrow 181$ & & & $\uparrow$ latency 180,194 & & & $\uparrow_{172,174,181,185,195}$ \\
\hline & & & & $\downarrow$ duration ${ }^{194}$ & & & \\
\hline & & & & $\uparrow$ duration $^{180}$ & & & \\
\hline
\end{tabular}

Abbreviations: TMS, transcranial magnetic stimulation; SICl, short-interval intracortical inhibition; ICF, intracortical facilitation; LICI, long-interval intracortical inhibition; IHI, interhemispheric inhibition; SAI, short-afferent inhibition; LAI, long-afferent inhibition; CMCT, central motor conduction time; $\mathrm{H}_{0}$, null result; ALS, amyotrophic lateral sclerosis; PD, Parkinson's disease; MS, multiple sclerosis; HD, Huntington's disease; $\uparrow$, increase; $\downarrow$, decrease. 
Table 6 Summary of pathology-related changes in repetitive transcranial magnetic stimulation-based measures of neurophysiology

\begin{tabular}{|c|c|c|c|c|c|}
\hline & \multicolumn{5}{|c|}{ Repetitive TMS } \\
\hline & $<\mathbf{I ~ H z}$ & $>\mathbf{I ~ H z}$ & cTBS & iTBS & PAS $_{\text {LTP }}$ \\
\hline $\mathrm{PD}$ & $\begin{array}{l}\text { PMd: } \uparrow \mathrm{SICl}^{54} \\
\text { SD: } \uparrow \mathrm{CSP}^{57} \\
\text { SD: } \uparrow \mathrm{SICl}^{57} \\
\text { MD: } \uparrow \mathrm{CSP}^{196}\end{array}$ & $\begin{array}{l}\mathrm{H}_{0}^{197} \\
\mathrm{PMd}: \mathrm{H}_{0}^{106} \\
\mathrm{SD}: \uparrow \mathrm{CSP}^{52} \\
\mathrm{SD}: \uparrow \mathrm{CSP}^{57} \\
\mathrm{SD}: \uparrow \mathrm{ICF}^{57} \\
\mathrm{MD}: \uparrow \mathrm{CSP}^{74,198} \\
\mathrm{MD}: \uparrow \mathrm{SICl}^{74} \\
\mathrm{MD}: \uparrow \mathrm{MT}^{74}\end{array}$ & $\mathrm{H}_{0}^{199}$ & $\mathrm{H}_{0}^{200}$ & $\begin{array}{l}\mathrm{H}_{0} \mathrm{MEP}^{201-203} \\
\mathrm{H}_{0} \mathrm{CSP}^{201} \\
\uparrow \mathrm{MEP}^{201,202,204} \\
\downarrow \mathrm{CSP}^{204} \\
\downarrow \mathrm{SICl}^{203} \\
\downarrow \mathrm{ICF}^{203}\end{array}$ \\
\hline HD & & $\begin{array}{l}\mathrm{H}_{0} \mathrm{MEPs}^{213} \\
\uparrow \mathrm{CSP}^{213}\end{array}$ & $\downarrow 205$ & & $\downarrow^{206}$ \\
\hline \multicolumn{6}{|l|}{ ALS } \\
\hline MS & & & & $\downarrow{\mathrm{H} / \mathrm{M}^{2}}^{2}$ & \\
\hline
\end{tabular}

Abbreviations: TMS, transcranial magnetic stimulation; cTBS, continuous theta burst stimulation; iTBS, intermittent theta burst stimulation; PAS LTP $_{\text {Paired associative }}$ stimulation, long-term potentiation induced; MEP, motor-evoked potential; CSP, cortical silent period; $\mathrm{H}_{0}$, null result; SICI, short-interval intracortical inhibition; ICF, intracortical facilitation; MT, motor threshold; PMd, dorsal premotor cortex; SD, single-day; MD, multi-day; H/M, Hoffman-reflex/M-wave; $\mathrm{H}_{0}$, null result; $\uparrow$, increased response compared with controls; $\downarrow$, decreased response compared with controls; ALS, amyotrophic lateral sclerosis; PD, Parkinson's disease; MS, multiple sclerosis; HD, Huntington's disease.

striatum. ${ }^{64}$ However, there are conflicting findings regarding whether stimulation over M1 translates to altered motor cortical excitability and/or alters the symptoms of PD; some studies showed increases in cortical excitability and improved symptoms, while others demonstrate no change following rTMS.

There is evidence that single-session rTMS over M1 representations can produce positive after-effects in PD. The first report of beneficial effects on motor behavior in PD applied $5 \mathrm{~Hz}$ rTMS over M1 and found improvement in simple and choice reaction time and pegboard task performance, ${ }^{65}$ which were not replicated in a subsequent study ${ }^{66}$ Further research demonstrated lasting positive effects on motor behavior in $\mathrm{PD}$ after similar stimulation. ${ }^{52}$ Specifically, 5 Hz rTMS over M1 prolonged the CSP, ${ }^{52}$ and improved symptoms of bradykinesia ${ }^{67}$ and aiming movements. ${ }^{68}$ Further study confirmed the benefits of rTMS over M1 by showing that both $0.5 \mathrm{~Hz}$ and $10 \mathrm{~Hz}$ rTMS resulted in improvement of motor symptoms. ${ }^{57}$ Specifically, Lefaucheur et al found that $10 \mathrm{~Hz}$ rTMS reduced contralateral bradykinesia, $0.5 \mathrm{~Hz}$ rTMS reduced bilateral rigidity and improved walking speed, and $10 \mathrm{~Hz}$ and $0.5 \mathrm{~Hz}$ rTMS prolonged the CSP, with $0.5 \mathrm{~Hz}$ rTMS increasing SICI and $10 \mathrm{~Hz}$ rTMS increasing ICF. Interestingly, $10 \mathrm{~Hz}$ rTMS over M1 has been shown to improve function in PD similarly to that of levodopa treatment transiently. ${ }^{69}$ Also, reductions in akinesia in the arm contralateral to the stimulation have been shown due to a single session of iTBS over $\mathrm{M}^{31}$ for those on and off medication. ${ }^{70}$ Speech, voice intensity, and fundamental frequency have shown improvements due to $15 \mathrm{~Hz}$ rTMS over the M1 mouth area in PD. These studies demonstrate the usefulness of rTMS over M1 representation for short-term normalization of intracortical circuitry, symptoms, and motor function commonly affected in PD.

There is also evidence of the potential for prolonged benefits of repeated sessions of rTMS over M1 in PD. 5 Hz rTMS applied over the lower and upper limb M1 representations once per day for 10 consecutive days resulted in improvement in total motor UPDRS, walking speed, and self-assessment scale for up to 1 month after rTMS. ${ }^{71}$ Similarly, $25 \mathrm{~Hz}$ rTMS over the leg and bilateral arm representations in M1 for 6 consecutive days showed improvements in total motor UPDRS, key-tapping, walking speed, and self-assessment scale across sessions that lasted for 1 month, with the greatest benefits with those in early PD. ${ }^{72}$ Three supplemental sessions of rTMS at monthly intervals following the initial treatment sessions were able to counteract transient decay of benefits. Additionally, rTMS over only the lower limb representation in $\mathrm{M}^{73}$ and with the combination of treadmill training has been shown to improve gait and other motor functions. ${ }^{74}$ A $5 \mathrm{~Hz}$ rTMS over the leg area in M1 followed by treadmill training for 12 sessions showed improved walking speeds, timed "up and go" measures, as well as normalized neurophysiological TMS measures (Tables 4-6). ${ }^{74}$ Similar improvements in gait along with overall UPDRS-III scores, visual analog scale, self-assessment score, and fingertapping task performance were observed after 3 consecutive days of only $10 \mathrm{~Hz}$ rTMS over M1 foot muscle representation. ${ }^{73}$ Daily rTMS showed no cumulative benefits compared with a single day, but could be effective in maintaining the observed improvements in motor behavior.

Although vastly different in the specific M1 targets, intensity, duration, frequency, and with or without the combination of behavioral or drug interventions, all of these 
studies demonstrate the potential usefulness of multi-session rTMS over M1 areas in improving motor function along with normalization of cortical excitability in PD.

\section{rTMS over the dorsal lateral prefrontal cortex}

The DLPFC is an important cortical target in the application of rTMS for the treatment of depression associated with PD. ${ }^{75-86}$ This concept of altering DLPFC excitability in individuals with $\mathrm{PD}$ originated from evidence for reduced blood oxygen level-dependent activation of the DLPFC suggesting abnormal frontal cortical activation in PD. ${ }^{87,88}$ Importantly, there is evidence of improvement in motor function (UPDRS scores) after multiple sessions of $10 \mathrm{~Hz}$ rTMS over the DLPFC. ${ }^{81}$ Additionally, transient reductions in punding (complex, repetitive, stereotyped behavior as a result of LID) have been observed after a single session of $1 \mathrm{~Hz}$ rTMS over the right DLPFC. ${ }^{89}$ Therefore, there is evidence that rTMS over the DLPFC in single and multiple sessions can improve depression, mood, and even motor behavior associated with PD.

\section{rTMS application over both MI and DLPFC}

Recent studies have combined rTMS over M1 and the DLPFC across multiple sessions in an attempt to address both motor and depression symptoms in individuals with PD, with varying success. ${ }^{90-92}$ Multiple sessions of $25 \mathrm{~Hz}$ rTMS over M1 and the DLFPC bilaterally showed significant improvements in times for execution in walking and complex hand movements lasting 1 month after treatment. ${ }^{90}$ Left M1 enhancements in MEPs were correlated with improvements in right hand bradykinesia after individual sessions, but overall motor improvement was not related to changes in M1 excitability. ${ }^{90}$ Interestingly, a recent study investigated the effects of using an H-shaped-coil (which induces deeper and wider magnetic fields) to administer rTMS over M1 and bilateral DLPFC (in that order) as an add-on treatment for motor symptoms of PD in individuals on medication. ${ }^{92}$ Multiple weekly sessions of $10 \mathrm{~Hz} \mathrm{H}$-coil rTMS is safe, producing no adverse effects, and is also potentially effective in improving motor function (UPDRS-III) when applied with conventional PD medication. Although the application of rTMS over multiple cortical loci during multiple sessions may confound the contribution of each cortical area to the observed effects, these studies demonstrate the potential application of rTMS to simultaneously modulate different striatocortical loops and improve cognitive and motor function in PD for a prolonged period after rTMS treatment.

\section{rTMS applied over the supplementary motor area}

There is evidence that SMA function is compromised in the progression of PD. ${ }^{93}$ Abnormal SMA activity has been associated with bradykinesia ${ }^{44}$ and other task-related paradigms, ${ }^{94}$ while others show overactivation of SMA in dyskinetic PD. ${ }^{62-95}$ The rationale for SMA as a target cortical location for application of rTMS is that input to the SMA largely originates from basal ganglia output. ${ }^{96}$ Additionally, basal ganglia to SMA motor pathways involved in self-paced voluntary movement control shows the greatest deficits in individuals with PD. ${ }^{93,97}$ Generally, there is more evidence for the beneficial effects of inhibitory $1 \mathrm{~Hz}$ rTMS compared with excitatory $(>1 \mathrm{~Hz})$ rTMS over SMA in both single and multiple sessions.

Single-session application of $1 \mathrm{~Hz}$ rTMS over SMA can reduce LID transiently, ${ }^{98}$ with no additional benefits after multiple sessions on the transient effects. ${ }^{99}$ Conversely, there is evidence that multi-session $1 \mathrm{~Hz}$ rTMS over SMA reduces LIDs for up to 24 hours after stimulation. ${ }^{100}$ Another recent randomized controlled trial involving individuals with $\mathrm{PD}$ showed that multiple sessions of $1 \mathrm{~Hz}$ rTMS over SMA improved motor function significantly (UPDRS), with the effects lasting for 20 weeks post stimulation. ${ }^{101}$ These studies provide evidence that SMA may be a promising locus for rTMS as a treatment for motor function, bradykinesia, and LID in the later stages of PD.

\section{rTMS over the premotor cortex}

Few studies have investigated the effects of rTMS over PMd, demonstrating slight changes in cortical excitability without clinically relevant measures. The reason for the PMd as a target cortical location is due to other studies showing that rTMS over PMd can modulate intracortical excitability in the downstream M1 in healthy individuals, ${ }^{102-104}$ and also there is abnormally increased activity of PMd in individuals with PD, which is thought to compensate for deficient activity from the basal ganglia to frontal lobe areas. ${ }^{45}$ One study in which $1 \mathrm{~Hz}$ rTMS over PMd was applied to early-stage, non-medicated individuals with PD found normalized (increased) SICI that lasted for a week after stimulation. ${ }^{54}$ One Hz rTMS over PMd was also found to prolong an abnormally shortened CSP in PD, without concurrent improvements in clinical measures. ${ }^{105}$ When higher frequency rTMS is applied over PMd (ie, $>1 \mathrm{~Hz}$ ) there are little to no beneficial effects. ${ }^{78,106}$ Therefore, studies on rTMS over the premotor cortex show little benefit in terms of motor function, despite modulation of neuro- 
physiological measures when inhibitory rTMS is applied. A potential explanation for this may be that the premotor cortex could not be as affected by neurodegeneration of the substantia nigra pars compacta due to less of an input source from the basal ganglia, whereas an area like SMA has been shown to have vital input from the basal ganglia and therefore would be more affected by degeneration of the striato-motor pathways. ${ }^{96}$ Additionally, the application of rTMS over SMA to treat the symptoms of PD may produce more beneficial results, since individuals with PD display the greatest deficits in the motor pathways between the basal ganglia and SMA, which are involved in self-paced voluntary movement control. ${ }^{93,97}$ However, further research is required to fully elucidate the potential clinical benefits of rTMS over each of these cortical areas.

\section{rTMS over the cerebellum}

Some studies have identified the cerebellum as a target for rTMS in the treatment of PD symptoms ${ }^{107,108}$ due to recent studies showing abnormalities (hyperexcitability) in the cerebellothalamocortical networks in PD and in LID. ${ }^{109-112}$ Koch et al demonstrated that a single-session cTBS applied over the lateral cerebellum decreased LID, while reducing SICI and increasing LICI in contralateral M1. In a follow-up study, multiple sessions of bilateral cerebellum cTBS showed persisting reductions in LID up to 6 weeks after rTMS treatment without improvements in motor function. ${ }^{107}$ Another study investigated the effects of a single session of $1 \mathrm{~Hz}$ rTMS over the right lateral cerebellum on motor performance of the upper limb in early-stage PD, and found that gross motor movements improved and fine motor movements worsened. ${ }^{108}$ These studies suggested that the cerebellum plays a role in motor symptoms and motor control in PD and offers a potential locus for rTMS as treatment, particularly with those experiencing LID.

A wealth of research has demonstrated the potential usefulness of rTMS as a treatment for psychological, cognitive, and motor symptoms, as well as normalization of the underlying abnormalities in cortical excitability associated with PD. Although there are conflicting findings, the preliminary positive findings of normalization of certain neurophysiological measures and improved functional outcomes warrant further study of rTMS as a treatment for PD. Potential promising avenues may be to explore the efficacy of multiple sessions of $1 \mathrm{~Hz}$ rTMS over particular cortical nodes (ie, SMA and M1) in combination with behavioral interventions such as gait, posture, and treadmill training, as well as upper limb movement training, such as bimanual training, depending on the particular motor deficits of individuals with PD. $1 \mathrm{~Hz}$ rTMS to either (or both) SMA or M1 may serve to normalize cortical excitability, and behavioral interventions may capitalize on the transient modulations of cortical excitability to provide enhanced improvements in motor ability and behavioral symptoms in PD.

\section{Huntington's disease}

Originating from disruption in basal ganglia functioning as the result of an expansion in the CAG triplet in the IT15 gene, ${ }^{113,114} \mathrm{HD}$ involves loss of GABA-ergic neurons in the striatum. ${ }^{113,115-117}$ This leads to reduced inhibition of the thalamus and subsequent overexcitation of the cortex. ${ }^{118}$ The ability of TMS to quantify levels of cortical excitability and inhibition make it a prime candidate for assessing the cortical impact of HD. The symptomology of HD includes motor (chorea, rigidity), cognitive (attentional deficits, perseveration), and psychiatric components (depression); ${ }^{114}$ however, TMS is primarily used to evaluate the motor aspect, as MEPs provide a quantifiable outcome measure.

\section{Assessment}

Various single-pulse and paired-pulse techniques have been used to assess the neurophysiology of HD, as well as disease progression. To date, due to numerous factors, such as sample size, stimulation parameters, experimental protocol, and heterogeneity between phenotypes, TMS has produced many conflicting results when assessing neurophysiology. A summary of these results can be seen in Tables 4-6. This section highlights some of the clinically relevant neurophysiology indexed by TMS.

Single-pulse TMS at a specific intensity produces decreased MEP amplitudes as compared with controls, which correlates with the severity of choreic movements. ${ }^{119}$ CSP has also been linked with the severity of choreic movements. When examined cross-sectionally, contradictory evidence has been presented, but a recent study correlated prolongation of the silent period with the severity of chorea. ${ }^{120}$ Longitudinally, over a 2-month follow-up, a decrease in CSP was shown to correlate with Unified Huntington's Disease Rating motor scores despite no differences at baseline. ${ }^{121,122}$ The final neurophysiological measure that translated to clinical relevance was short-afferent inhibition; decreased short-afferent inhibition compared with controls negatively correlated with the expected age of symptom onset. ${ }^{123}$ Future work aiming to assess the underlying neurophysiology of HD and potentially extract potential biomarkers for disease progression should, to the best of ability with such a rare 
disease, expand to greater sample sizes, and more tightly control for external factors such as number of CAG repeats, stage of disease, and experimental protocols.

\section{Treatment}

There is little evidence supporting or negating the efficacy of a TMS-based treatment for HD. Work in animal models using repetitive cortical stimulation has shown great promise in slowing, and even reversing, disease progression; however, this has not been supported with human work. Preliminary studies investigating the influence of rTMS on choreic movements in HD patients have produced conflicting results. These studies targeted SMA, likely due to its role in voluntary movement production and as a cortical region affected by basal ganglia output. The first study administered a single session of $1 \mathrm{~Hz}$ rTMS over SMA, compared with a single session of $5 \mathrm{~Hz}$ rTMS and sham stimulation. The authors found that $1 \mathrm{~Hz}$ rTMS led to a reduction in choreic movements for 30 minutes. ${ }^{124} \mathrm{~A}$ similar study administered $1 \mathrm{~Hz}$ rTMS over the SMA for seven sessions on separate days, reporting no influence of rTMS. ${ }^{125}$ These contradictory results question the efficacy of rTMS, specifically over the SMA, in the treatment of HD. However, both studies included a limited number of participants (four and two, respectively), which may influence the results. Further work should attempt to recruit a greater number of participants in specific stages of HD to determine the overall effect of rTMS as a potential adjunctive treatment. Additionally, application of rTMS over different cortical regions, such as M1 and primary somatosensory cortex, which are heavily involved in motor production and voluntary movement, may yield more success. The exclusive application of rTMS to cortical sites in individuals with HD will likely have mild impact, at best, on choreic movement; however, if future work defines this as a promising avenue, when used in conjunction with various other treatments, a more global impact on reducing symptomology may be accomplished.

\section{Amyotrophic lateral sclerosis}

ALS is a progressive neurodegenerative disease with no known cure, no effective therapies, and likely has multiple causes. ALS affects the upper and lower motor neurons in the motor cortex, brain stem, and spinal cord. ${ }^{126}$ Glutamatemediated excitotoxicity is a proposed mechanism underlying the pathology of the disease. ${ }^{127}$ The neural circuitry underlying ALS can be noninvasively probed using TMS, ${ }^{128-141}$ and a number of studies have investigated the potential of rTMS as a treatment for ALS, mainly by slowing the progression of the disease. ${ }^{142-149}$

\section{Assessment}

Several studies have investigated the potential mechanisms underlying the neural degeneration in ALS, with common and conflicting findings. Measures of cortical excitability abnormalities in ALS are summarized in Tables 4-6. Some of these studies conflict regarding the usefulness of TMS as a method of ALS diagnosis, ${ }^{150,151}$ with most confirming its usefulness, particularly in early and subclinical ALS when it is combined with clinical and conventional neurophysiological recordings. ${ }^{128,130,132,141,152-155}$ The reasons for the conflicting neurophysiological findings are unclear; however, some could be due to disease progression, duration, medication status, and/or the relatively low sample sizes and methods of TMS used to acquire neurophysiological measures. Generally, M1 hyperexcitability is found in intracortical mechanisms along with abnormal interhemispheric interactions, with little consistent data on the responsiveness to rTMS protocols. The evidence suggests that normal or reduced MT, increased MEP amplitudes, and reduced SICI are associated with general hyperexcitability and glutamateinduced neurotoxicity, ${ }^{133,156-158}$ which may be indicative of an anterograde transsynaptic mechanism of central and peripheral neural degeneration. ${ }^{126,159,160}$ Finally, a few studies have reported that MT, MEP amplitudes, CMCT, and transcallosal inhibition can correlate with disease duration, progression, ${ }^{130,132,141,161,162}$ functional deficits, ${ }^{140,141,161}$ and fatigue; ${ }^{163}$ however, not all work in this area consistently reports these changes. ${ }^{164}$

It has been suggested that the sensitivity of assessment of upper motor neuron dysfunction may be enhanced by testing orofacial muscle representation (probing the corticobulbar tracts) along with the traditional limb muscles. ${ }^{165-167}$ Additionally, the identification of ALS may be improved by using the triple stimulation technique. ${ }^{168}$ The triple stimulation technique uses a single TMS pulse followed by two peripheral electrical stimulations (wrist and Erb's point) in order to understand the differential cortical and peripheral contributions to the generated motor response. For this reason, several studies have reported that the triple stimulation technique provides improved sensitivity of TMS to detect upper and lower motor neuron dysfunction. ${ }^{169-171}$

\section{Treatment}

There have been a number of studies investigating the effects of rTMS application over M1 in an attempt to treat the motor 
symptoms of ALS. ${ }^{142-149}$ It is difficult to form generalized conclusions from this body of work owing to the heterogeneity of the rTMS employed; these protocols differed in terms of the frequency, duration, and dose of stimulation delivered. ${ }^{149}$ Some studies show improvements in symptoms, behavior, and normalization of cortical excitability due to rTMS. However, other work does not show consistent improvements in reduction of the progression of ALS. ${ }^{143,146,149}$ In general, most of the studies used low frequency rTMS or cTBS over M1 in order to normalize (decrease) the hyperexcitability most commonly reported in ALS. The following section summarizes the current state of the literature regarding the use of rTMS to treat the symptoms and abnormal cortical excitability seen in ALS.

One of the first studies investigating rTMS in ALS examined disease progression. ${ }^{143}$ Two individuals with ALS received $1 \mathrm{~Hz}$ rTMS and two individuals with ALS (both taking riluzole) received $20 \mathrm{~Hz}$ rTMS over the first dorsal interosseous (FDI) M1 representation bilaterally. One $\mathrm{Hz}$ rTMS was applied over each M1 on weekdays over 2 weeks, every 4 months. The $20 \mathrm{~Hz}$ rTMS was applied over each M1 in short trains for 8 consecutive days every month. The Norris rating scale and the Medical Research Council scale were used to index disease progression before and during rTMS treatment. Although all of the study participants tolerated the rTMS treatment well, they continued to deteriorate clinically. Progression was slightly slower (no significance) for the individuals who received $1 \mathrm{~Hz}$ rTMS compared with the time period before rTMS, whereas the opposite was true for the individuals who were treated with $20 \mathrm{~Hz}$ rTMS. The authors concluded that low frequency rTMS may give a new therapeutic perspective to ALS, but given that there was not a control group who received sham stimulation, it is unclear that the slowed progression was due to brain stimulation. ${ }^{143}$

A double-blind, placebo-controlled study investigated the potential beneficial effects of cTBS over M1 FDI representation on motor function in individuals with ALS (all on riluzole). ${ }^{144}$ Individuals with ALS received either cTBS $(n=7)$ or sham stimulation $(n=8)$ over M1 in both hemispheres on 5 consecutive days, once a month for 6 consecutive months. Both individuals who received cTBS and sham stimulation showed continued declines in motor function as measured by the revised ALS functional rating scale (ALSFRS-R); however, the cTBS group showed a slight but significant slowing in the rate of deterioration. ${ }^{144}$

Another study suggested that $5 \mathrm{~Hz}$ rTMS over M1 may transiently improve motor function and quality of life in
ALS. ${ }^{145}$ Specifically, rTMS was applied over the thenar muscle representations with a figure-of- 8 coil and over tibialis anterior representation with a circular coil in both hemispheres. rTMS was delivered for 5 consecutive days per week for 2 weeks to ten individuals with ALS (all taking riluzole; rTMS $n=5$, sham $n=5$ ). Those with ALS who received rTMS treatment improved in quality of life scores, maximum voluntary isometric contraction, and isokinetic average power immediately after the 2 weeks of stimulation treatment, with no significant changes at a 2-week follow-up after rTMS treatment had stopped. However, the authors suggest that these findings should be interpreted with caution due to the small number of patients and lack of a doubleblind protocol.

Di Lazzaro et al performed another double-blind, placebocontrolled rTMS treatment intervention in 20 people with ALS (all taking riluzole) randomly assigned to cTBS or sham stimulation over a prolonged period of time. cTBS over bilateral FDI M1 representation was performed for 5 consecutive days, every month for 1 year. It was found that ALSFRS-R score deterioration was not different between cTBS and sham stimulation. It was concluded that a larger and prolonged randomized controlled trial with ALS patients seemed unjustified, at least for the advanced stages of the disease. ${ }^{146}$ Similarly, a separate study, also by Di Lazzaro et al, ${ }^{148}$ investigated the effects of further prolonged treatment of rTMS over M1 on ALS disease progression. Two ALS patients (both on riluzole) participated in the study with the same stimulation protocol as in Di Lazzaro et al, ${ }^{146}$ except over 2 years. The other ALS patient received chronic epidural M1 stimulation. There was a slight reduction in ALSFRS-R score deterioration rate in the individual who received cTBS treatment compared with epidural stimulation, with the most pronounced slowing 1 year after cTBS treatment began. In the patient who received cTBS, MEPs and CMCT were acquired from the biceps, FDI, and tibialis anterior muscles before, throughout, and after the treatment time. There were no consistent changes in the TMS-acquired measures across the cTBS treatment period. Once again, interpretation of these findings must be made with caution due to only one patient receiving cTBS treatment. ${ }^{148}$

Although there were some promising preliminary findings, recent studies on the treatment of ALS with rTMS over M1 protocols have shown at best slight and at worse no beneficial effects. Any improvements that were noted occurred via slowing of progression of the disease. However, given that there are no effective treatments for people with ALS, further work is warranted to investigate varying the 
specific duration, technique, and frequency of stimulation that in combination may enable disease progression to be slowed. ${ }^{147,149}$ For example, paired associated stimulation may be a potential future avenue to modulate abnormal nervous system activation in ALS. The pairing of peripheral nerve stimulation (sending a somatosensory signal to the cortex via the spinal cord) with TMS pulse over M1 hand representation (eg, abductor pollicis brevis) repetitively may positively modulate the abnormal excitability in ALS.

\section{Multiple sclerosis}

MS is an idiopathic inflammatory disorder of the central nervous system, ${ }^{172}$ and is the major cause of nontraumatic disability in young adults. ${ }^{173} \mathrm{MS}$ is characterized by demyelination of the periventricular white matter, brain stem, and spinal cord, ${ }^{174}$ but axonal degeneration is sometimes observed early in the disease course. ${ }^{172}$ Individual variability is very prevalent in MS. The majority of patients begin in a relapsing stage of the disease (relapse-remitting MS) followed after several years by a secondary progressive stage. ${ }^{175,176}$ Some individuals show a primary progressive pattern from disease onset; however, the rate of progression is different per individual. ${ }^{177}$ The Expanded Disability Status Scale (EDSS) is the major scale used to measure clinical disability in individuals with MS. Due to limitations such as interrater reliability, low sensitivity to clinical change, and being an ordinal scale, its effectiveness as a marker of clinical progression is limited. ${ }^{173}$ This highlights the need for a more reliable neurobiological marker.

\section{Assessment}

Multiple electrophysiological tests have been employed for the diagnosis of MS, and most of the studies indicate that these are of little or no value. ${ }^{178,179}$ Magnetic resonance images of the brain are the major contributor to diagnosis of MS, but are hindered by low specificity and negative results. Recently, the use of TMS-based evoked potentials have evolved into a marker of disease progression ${ }^{180}$ and have been studied as a surrogate marker of clinical progression in MS. ${ }^{172-174,181-183}$ Schmierer et al found that abnormal transcallosal inhibition and CMCT significantly correlated with EDSS scores ${ }^{180}$ $(P<0.01)$, and correlations have been noted between EDSS scores and MEP latencies, MTs, and short latency ICF. ${ }^{173}$ Importantly, TMS-evoked potentials in individuals with MS correlate with disability as indexed by the EDSS. ${ }^{172}$ Together, these results suggest that the combination of different TMS measures can provide objective data that assist in estimating disease progression in MS.

\section{Treatment}

Individuals with MS usually suffer from mild to severe spasticity. ${ }^{184}$ To date, the pharmacological treatment of spasticity has had limited success, and causes multiple side effects, such as drowsiness, muscle weakness, and cognitive disturbances. ${ }^{28,138,139,180,181,185-207}$ Hyperexcitability of the stretch reflex is considered a possible mechanism of spasticity, due to easily activated stretch reflexes in antagonistic muscle. This can impede the initiation of agonist movement ${ }^{207}$ and highlights the need for nonpharmacological treatment ${ }^{185}$ such as rTMS.

Centonze et $\mathrm{al}^{208}$ used $5 \mathrm{~Hz}$ and $1 \mathrm{~Hz}$ rTMS to attempt to modulate the hyperexcitability of the stretch reflex arc in 19 individuals in the remitting stage of relapse-remitting MS. A single session of $5 \mathrm{~Hz}$ rTMS over the leg area of M1 increased corticospinal excitability and decreased the H/M amplitude ratio of the soleus $\mathrm{H}$ reflex, implicating decreased antagonist muscle tone, whereas inhibitory rTMS increased the $\mathrm{H} / \mathrm{M}$ amplitude ratio. Yet single sessions of stimulation did not have a clear clinical effect on spasticity. Centonze et al performed another experiment where $5 \mathrm{~Hz}$ rTMS was applied once daily on 5 consecutive days for 2 weeks. Significant improvement of lower limb spasticity was observed. Long-term reduction of $\mathrm{H} / \mathrm{M}$ amplitude was observed, leading to the conclusion that rTMS can contribute to reducing spasticity in individuals with MS. ${ }^{208}$

Mori et al investigated whether iTBS is an effective modulator of lower limb spasticity in individuals with MS. The protocol consisted of a 2 -week period where 20 patients received daily sessions of iTBS or sham stimulation. Outcome measures consisted of the $\mathrm{H} / \mathrm{M}$ amplitude ratio of the soleus $\mathrm{H}$ reflex and the Modified Ashworth Scale for spasticity. iTBS was delivered over the M1 corresponding to the soleus muscle. Significant improvement was shown during the active iTBS protocol through a reduction of $\mathrm{H} / \mathrm{M}$ amplitude and Modified Ashworth Scale scores 1 week after stimulation. No changes were shown with sham stimulation. Changes persisted up to 2 weeks after the end of the stimulation protocols. This shows promise for iTBS to modulate spasticity and improve quality of life in individuals with $\mathrm{MS}^{207}$

Another approach to the management of spasticity in individuals with MS is to pair exercise therapy with iTBS. iTBS was delivered over the site of M1 corresponding to soleus for 2 weeks. Exercise alone did not result in a significant improvement in spasticity; however, iTBS alone led to a reduction in spasticity (as measured by the Modified Ashworth Scale). iTBS plus exercise improved measures of spasticity, function, and quality of life. Prior exercise studies 
have shown positive effects in 8 weeks to 6 months, leading to the assumption that priming the motor cortex through iTBS anticipates the neuronal adaptations underlying exercise in individuals with MS. ${ }^{209}$

The effects of $5 \mathrm{~Hz}$ excitatory rTMS over M1 in individuals with MS and cerebellar impairment have also been considered. MS participants $(n=8)$ and healthy controls $(n=7)$ completed a nine-hole pegboard task five times prior to rTMS, five times immediately after rTMS, and 10 minutes and 20 minutes after brain stimulation. rTMS was delivered over the site of M1 corresponding to the FDI muscle. Significant reduction in the time required to complete this task was observed in the individuals with MS. There was no significant improvement observed in the healthy controls. Further investigations are needed to verify whether rTMS can induce lasting clinical improvement in individuals with MS who have cerebellar symptoms. ${ }^{210}$

rTMS over M1 is a hopeful aid for symptoms of spasticity associated with MS when paired with exercise or as its own intervention. ${ }^{185,207,209,210}$ As a variety of symptoms are associated with MS, rTMS may be used in the future as a component of a more complete treatment protocol to specifically target spasticity, with other avenues alleviating different symptoms. TMS has the potential to be a successful method of marking disease progression; however, future studies are needed to validate these findings as well as to determine whether they may extend benefit to other motor symptoms associated with MS.

\section{Conclusion}

As more research emerges, TMS is proving to be a promising technique in assessing the progression of and underlying neurophysiology of various movement disorders. The applicability of rTMS as a treatment is pathology-specific, with varying success depending on disease characteristics and the parameters of rTMS treatment. Further research and standardized administration procedures are warranted in order to further elucidate the promising preliminary work showing that altering cortical excitability with rTMS may lessen symptoms and normalize neurophysiological excitability in individuals with movement disorders.

\section{Disclosure}

The authors report no conflicts of interest in this work.

\section{References}

1. Di Lazzaro V, Ziemann U. The contribution of transcranial magnetic stimulation in the functional evaluation of microcircuits in human motor cortex. Front Neural Circuits. 2013;7:18.
2. Rossini PM, Barker AT, Berardelli A, et al. Non-invasive electrical and magnetic stimulation of the brain, spinal cord and roots: basic principles and procedures for routine clinical application. Report of an IFCN committee. Electroencephalograph Clin Neurophysiol. 1994;91(2):79-92.

3. Rothwell JC, Hallett M, Berardelli A, Eisen A, Rossini P, Paulus W. Magnetic stimulation: motor evoked potentials. The International Federation of Clinical Neurophysiology. Electroencephalogr Clin Neurophysiol Suppl. 1999;52:97-103.

4. Devanne H, Lavoie BA, Capaday C. Input-output properties and gain changes in the human corticospinal pathway. Exp Brain Res. 1997;114: 329-338.

5. Ridding MC, Rothwell JC. Stimulus/response curves as a method of measuring motor cortical excitability in man. Electroencephalogr Clin Neurophysiol. 1997;105:340-344.

6. Hess CW, Mills KR, Murray NM. Responses in small hand muscles from magnetic stimulation of the human brain. $J$ Physiol. 1987;388: 397-419.

7. Levy WJ, Amassian VE, Schmid UD, Jungreis C. Mapping of motor cortex gyral sites non-invasively by transcranial magnetic stimulation in normal subjects and patients. Electroencephalogr Clin Neurophysiol. 1991;43:51-75.

8. Wassermann EM, McShane LM, Hallett M, Cohen LG. Noninvasive mapping of muscle representations in human motor cortex. Electroencephalogr Clin Neurophysiol. 1992;85:1-8.

9. Wilson SA, Thickbroom GW, Mastaglia FL. Transcranial magnetic stimulation mapping of the motor cortex in normal subjects: the representation of two intrinsic hand muscles. J Neurol Sci. 1993;18:134-144.

10. Mortifee P, Stewart H, Schulzer M, Eisen A. Reliability of transcranial magnetic stimulation for mapping the human motor cortex. Electroencephalogr Clin Neurophysiol. 1994;93:131-137.

11. Thickbroom GW, Sammut R, Mastaglia FL. Magnetic stimulation mapping of motor cortex: factors contributing to map area. Electroencephalogr Clin Neurophysiol. 1998;109:79-84.

12. Thickbroom GW, Byrnes ML, Mastaglia FL. A model of the effect of MEP amplitude variation on the accuracy of TMS mapping. Clin Neurophysiol. 1999;110:941-943.

13. Thickbroom G, Byrnes M. Corticomotor organisation and motor function in multiple sclerosis. J Neurol. 2005;252:765-771.

14. Pearce AJ, Thickbroom GW, Byrnes ML, Mastaglia FL. Functional reorganisation of the corticomotor projection to the hand in skilled racquet players. Exp Brain Res. 2000;130:238-243.

15. Thielscher A, Kammer T. Linking physics with physiology in TMS: a sphere field model to determine the cortical stimulation site in TMS. Neuroimage. 2002;17:1117-1130.

16. Uy J, Ridding MC, Miles TS. Stability of maps of human motor cortex made with transcranial magnetic stimulation. Brain Topogr. 2002;14:293-297.

17. Chen R, Lozano AM, Ashby P. Mechanism of the silent period following transcranial magnetic stimulation. Evidence from epidural recordings. Exp Brain Res. 1999;128:539-542.

18. Fuhr P, Agostino R, Hallett M. Spinal motor neuron excitability during the silent period after cortical stimulation. Electroencephalogr Clin Neurophysiol. 1991;81:257-262.

19. Ingulleri M, Berardelli A, Cauccu G, Marfredi M. Silent period evoked by transcranial stimulation of the human cortex and cervicomedullary junction. J Physiol. 1993;466:521-534.

20. Kujirai T, Caramia MD, Rothwell JC, et al. Corticocortical inhibition in the human motor cortex. J Physiol. 1993;471:501-519.

21. Valls-Sole J, Pascual-Leone A, Wassermann EM, Hallett M. Human motor evoked responses to paired transcranial magnetic stimulation. Electroencephalogr Clin Neurophysiol. 1992;85:355-364.

22. Wassermann EM, Samii A, Mercuri B, et al. Responses to paired transcranial magnetic stimuli in resting, active, recently activated muscle. Exp Brain Res. 1996;109:158-163.

23. Mills KR, Nithi KA. Peripheral and central motor conduction in amyotrophic lateral sclerosis. J Neurol Sci. 1998;159:82-87. 
24. Chen R. Interactions between inhibitory and excitatory circuits in the human motor cortex. Exp Brain Res. 2004;154:1-10.

25. Manganotti P, Zanette G, Bonato C, Tinazzi M, Polo A, Fiaschi A. Crossed and direct effects of digital nerves stimulation on motor evoked potential: a study with magnetic brain stimulation. Electroencephalogr Clin Neurophysiol. 1997;105:280-289.

26. Tokimura H, Ridding MC, Tokimura Y, Amassian VE, Rothwell JC. Short latency facilitation between pairs of threshold magnetic stimuli applied to human motor cortex. Electroencephalogr Clin Neurophysiol. 1996;101:263-272.

27. Sailer A, Molnar GF, Cunic DI, Chen R. Effects of peripheral sensory input on cortical inhibition in humans. $J$ Physiol. 2002;544 Pt 2: 617-629.

28. Sailer A, Molnar GF, Paradiso G, Gunraj CA, Lang AE, Chen R. Short and long latency afferent inhibition in Parkinson's disease. Brain. 2003;126 Pt 8:1883-1894.

29. Stefan K, Kunesch E, Cohen LG, Benecke R, Classen J. Induction of plasticity in the human motor cortex by paired associative stimulation. Brain. 2000;123 Pt 3:572-584.

30. Pascual-Leone A, Tommas JM, Keenan J, Canete C, Catala MD. Study and modulation of human cortical excitability with transcranial magnetic stimulation. J Clin Neurophysiol. 1998;15:333-343.

31. Huang YZ, Edwards MJ, Rounis E, Bhatia KP, Rothwell JC. Theta burst stimulation of the human motor cortex. Neuron. 2005;45:201-206.

32. Huang YZ, Rothwell JC, Lu CS, et al. The effect of continuous theta burst stimulation over premotor cortex on circuits in primary motor cortex and spinal cord. Clin Neurophysiol. 2009;120:796-801.

33. Hamada M, Murase N, Hasan A, Balaratnam M, Rothwell JC. The role of interneuron networks in driving human motor cortical plasticity. Cereb Cortex. 2013;23:1593-1605.

34. Suppa A, Ortu E, Zafar N, et al. Theta burst stimulation induces aftereffects on contralateral primary motor cortex excitability in humans. J Physiol. 2008;18 Pt 1:84489-184500.

35. Neva JL, Vesia M, Singh AM, Staines WR. Modulation of left primary motor cortex excitability after bimanual training and intermittent theta burst stimulation to left dorsal premotor cortex. Behav Brain Res. 2014;261:289-296.

36. Legon W, Dionne JK, Staines WR. Continuous theta burst stimulation of the supplementary motor area: effect upon perception and somatosensory and motor evoked potentials. Brain Stimul. 2013;6:877-883.

37. Stinear CM, Barber PA, Coxon JP, Verryt TS, Acharya PP, Byblow WD. Repetitive stimulation of premotor cortex affects primary motor cortex excitability and movement preparation. Brain Stimul. 2009;2:152-162.

38. Premji A, Rai N, Nelson A. Area 5 influences excitability within the primary motor cortex in humans. PLoS One. 2011;6:e20023.

39. Arasanz CP, Staines WR, Roy EA, Schweizer TA. The cerebellum and its role in word generation: a cTBS study. Cortex. 2012;48:718-724.

40. Bolton DA, Staines WR. Transient inhibition of the dorsolateral prefrontal cortex disrupts attention-based modulation of tactile stimuli at early stages of somatosensory processing. Neuropsychologia. 2011;49: 1928-1937.

41. George MS, Post RM. Daily left prefrontal repetitive transcranial magnetic stimulation for acute treatment of medication-resistant depression. Am J Psychiatry. 2011;168:356-364.

42. Huang YZ, Rothwell JC, Edwards MJ, Chen RS. Effect of physiological activity on an NMDA-dependent form of cortical plasticity in human. Cereb Cortex. 2008;18:563-570.

43. Lang AE, Lozano AM. Parkinson's disease. First of two parts. $N$ Engl J Med. 1998;339:1044-1053.

44. Haslinger B, Erhard P, Ka N, et al. Event-related functional magnetic resonance imaging in Parkinson's disease before and after levodopa. Brain. 2001;124 Pt 3:558-570.

45. Sabatini U, Boulanouar K, Fabre N, Martin F. Cortical motor reorganization in akinetic patients with Parkinson's disease. A functional MRI study. Brain. 2000;123 Pt 2:394-403.

46. Berardelli A, Rothwell JC, Thompson PD, Hallett M. Pathophysiology of bradykinesia in Parkinson's disease. Brain. 2001;124:2131-2146.
47. Cantello R, Gianelli M, Bettucci D, Civardi C, De Angelis M, Mutani R. Parkinson's disease rigidity: magnetic motor evoked potentials in a small hand muscle. Neurology. 1991;41:1449-1456.

48. Ellaway P, Davey N, Maskill D, Dick J. The relation between bradykinesia and excitability of the motor cortex assessed using transcranial magnetic stimulation in normal and parkinsonian subjects. Electroencephalogr Clin Neurophysiol. 1995;97:169-178.

49. Ridding M, Inzelberg R, Rothwell J. Changes in excitability of motor cortical circuitry in patients with Parkinsons disease. Ann Neurol. 1995;37:181-188.

50. Filippi MM, Oliveri M, Pasqualetti P, et al. Effects of motor imagery on motor cortical output topography in Parkinson's disease. Neurology. 2001;57:55-61.

51. Valls-Solé J, Pascual-Leone A, Brasil-Neto J, Cammarota A, McShane L, Hallett M. Abnormal facilitation of the response to transcranial magnetic stimulation in patients with Parkinson's disease. Neurology. 1994;44:735-741.

52. Siebner HR, Mentschel C, Auer C, Lehner C, Conrad B. Repetitive transcranial magnetic stimulation causes a short-term increase in the duration of the cortical silent period in patients with Parkinson's disease. Neurosci Lett. 2000;284:147-150.

53. Priori A, Berardelli A, Inghilleri M. Pharmacological changes in the silent period after transcranial brain stimulation in normal subjects, patients with Parkinson's disease and drug-induced parkinsonism. Brain. 1994:317-323.

54. Buhmann C, Gorsler A, Bäumer T, et al. Abnormal excitability of premotor-motor connections in de novo Parkinson's disease. Brain. 2004;127 Pt 12:2732-2746.

55. Berardelli A, Abbruzzese G, Chen R, et al. Consensus paper on short-interval intracortical inhibition and other transcranial magnetic stimulation intracortical paradigms in movement disorders. Brain Stimul. 2008;1:183-191.

56. Bareš M, Kaňovský P. Intracortical inhibition and facilitation are impaired in patients with early Parkinson's disease: a paired TMS study. Eur J Neurol. 2003;10:385-389.

57. Lefaucheur J-P, Drouot X, Von Raison F, Ménard-Lefaucheur I, Cesaro P, Nguyen J-P. Improvement of motor performance and modulation of cortical excitability by repetitive transcranial magnetic stimulation of the motor cortex in Parkinson's disease. Clin Neurophysiol. 2004;115: 2530-2541.

58. Dauper J, Peschel T, Schrader C, Kohlmetz C, Joppich G, Nager W. Effects of subthalamic nucleus (STN) stimulation on motor cortex excitability. Neurology. 2002;59:700-706.

59. Berardelli A, Rona S, Inghilleri M, Manfredi M. Cortical inhibition in Parkinson's disease. A study with paired magnetic stimulation. Brain. 1996;119 Pt 1:71-77.

60. Wu AD, Petzinger GM, Lin C-HJ, Kung M, Fisher B. Asymmetric corticomotor excitability correlations in early Parkinson's disease. Mov Disord. 2007;22:1587-1593.

61. Grafton ST. Contributions of functional imaging to understanding parkinsonian symptoms. Curr Opin Neurobiol. 2004;14:715-719.

62. Rascol O, Sabatini U, Brefel C, et al. Cortical motor overactivation in parkinsonian patients with L-dopa-induced peak-dose dyskinesia. Brain. 1998;121 Pt 3:527-533.

63. Thobois S, Jahanshahi M, Pinto S, Frackowiak R, Limousin-Dowsey P. PET and SPECT functional imaging studies in Parkinsonian syndromes: from the lesion to its consequences. Neuroimage. 2004;23:1-16.

64. Strafella AP, Paus T, Fraraccio M, Dagher A. Striatal dopamine release induced by repetitive transcranial magnetic stimulation of the human motor cortex. Brain. 2003;126 Pt 12:2609-2615.

65. Pascual-Leone A, Valls-Solé J, Brasil-Neto J, Cammarota A, Grafman J, Hallett M. Akinesia in Parkinson's disease. II. Effects of subthreshold repetitive transcranial motor cortex stimulation. Neurology. 1994;44:892-898.

66. Ghabra M, Hallet M, Wassermann E. Simultaneous repetitive transcranial magnetic stimulation does not speed fine movement in PD. Neurology. 1999;52:768-770. 
67. Siebner HR, Rossmeier C, Mentschel C, Peinemann A, Conrad B. Short-term motor improvement after sub-threshold 5 - $\mathrm{Hz}$ repetitive transcranial magnetic stimulation of the primary motor hand area in Parkinson's disease. J Neurol Sci. 2000;178:91-94.

68. Siebner H, Mentschel C, Auer C, Conrad B. Repetitive transcranial magnetic stimulation has a beneficial efffect on bradykinesia in Parkinson's disease. Neuroreport. 1999;10:589-594.

69. Börnke C, Schulte T, Przuntek H, Müller T. Clinical effects of repetitive transcranial magnetic stimulation versus acute levodopa challenge in Parkinson's disease. J Neural Transm Suppl. 2004;68:61-67.

70. Degardin A, Devos D, Defebvre L, et al. Effect of intermittent theta-burst stimulation on akinesia and sensorimotor integration in patients with Parkinson's disease. Eur J Neurosci. 2012;36: 2669-2678.

71. Khedr EM, Farweez HM, Islam H. Therapeutic effect of repetitive transcranial magnetic stimulation on motor function in Parkinson's disease patients. Eur J Neurol. 2003;10:567-572.

72. Khedr EM, Rothwell JC, Shawky OA, Ahmed MA, Hamdy A. Effect of daily repetitive transcranial magnetic stimulation on motor performance in Parkinson's disease. Mov Disord. 2006;21:2201-2205.

73. Maruo T, Hosomi K, Shimokawa T, et al. High-frequency repetitive transcranial magnetic stimulation over the primary foot motor area in Parkinson's disease. Brain Stimul. 2013;6:884-891.

74. Yang Y-R, Tseng C-Y, Chiou S-Y, et al. Combination of rTMS and treadmill training modulates corticomotor inhibition and improves walking in Parkinson disease: a randomized trial. Neurorehabil Neural Repair. 2013;27:79-86.

75. Shimamoto H, Takasaki K, Shigemori M, Imaizumi T, Ayabe M, Shoji $\mathrm{H}$. Therapeutic effect and mechanism of repetitive transcranial magnetic stimulation in Parkinson's disease. J Neurol. 2001;248 Suppl 3:48-51.

76. Ikeguchi M, Touge T, Nishiyama Y, Takeuchi H, Kuriyama S, Ohkawa M. Effects of successive repetitive transcranial magnetic stimulation on motor performances and brain perfusion in idiopathic Parkinson's disease. J Neurol Sci. 2003;209:41-46.

77. Kim JY, Chung EJ, Lee WY, et al. Therapeutic effect of repetitive transcranial magnetic stimulation in Parkinson's disease: analysis of [11C] raclopride PET study. Mov Disord. 2008;23:207-211.

78. Sedlácková S, Rektorová I, Srovnalová H, Rektor I. Effect of high frequency repetitive transcranial magnetic stimulation on reaction time, clinical features and cognitive functions in patients with Parkinson's disease. J Neural Transm. 2009;116:1093-1101.

79. Pal E, Nagy F, Aschermann Z, Balazs E, Kovacs N. The impact of left prefrontal repetitive transcranial magnetic stimulation on depression in Parkinson's disease: a randomized, double-blind, placebo-controlled study. Mov Disord. 2010;25:2311-2317.

80. Srovnalova H, Marecek R, Kubikova R, Rektorova I. The role of the right dorsolateral prefrontal cortex in the Tower of London task performance: repetitive transcranial magnetic stimulation study in patients with Parkinson's disease. Exp Brain Res. 2012;223:251-257.

81. Fregni F, Santos CM, Myczkowski ML, et al. Repetitive transcranial magnetic stimulation is as effective as fluoxetine in the treatment of depression in patients with Parkinson's disease. J Neurol Neurosurg Psychiatry. 2004;75:1171-1174.

82. Fregni F, Ono CR, Santos CM, et al. Effects of antidepressant treatment with rTMS and fluoxetine on brain perfusion in PD. Neurology. 2006;66: 1629-1637.

83. Cardoso EF, Fregni F, Martins Maia F, et al. rTMS treatment for depression in Parkinson's disease increases BOLD responses in the left prefrontal cortex. Int J Neuropsychopharmacol. 2008;11: $173-183$.

84. Del Olmo MF, Bello O, Cudeiro J. Transcranial magnetic stimulation over dorsolateral prefrontal cortex in Parkinson's disease. Clin Neurophysiol. 2007;118:131-139.

85. Dias A, Barbosa E. Effects of repetitive transcranial magnetic stimulation on voice and speech in Parkinson's disease. Acta Neurol Scand. 2006;113:92-99.
86. Dragasevic N, Potrebić A, Damjanović A, Stefanova E, Kostić VS. Therapeutic efficacy of bilateral prefrontal slow repetitive transcranial magnetic stimulation in depressed patients with Parkinson's disease: an open study. Mov Disord. 2002;17:528-532.

87. Buhmann C. Pharmacologically modulated fMRI - cortical responsiveness to levodopa in drug-naive hemiparkinsonian patients. Brain. 2003;126:451-461.

88. Dirnberger G, Frith CD, Jahanshahi M. Executive dysfunction in Parkinson's disease is associated with altered pallidal-frontal processing. Neuroimage. 2005;25:588-599.

89. Nardone R, De Blasi P, Höller Y, et al. Repetitive transcranial magnetic stimulation transiently reduces punding in Parkinson's disease: a preliminary study. J Neural Transm. 2014;121:267-274.

90. Lomarev MP, Kanchana S, Bara-Jimenez W, Iyer M, Wassermann EM, Hallett M. Placebo-controlled study of rTMS for the treatment of Parkinson's disease. Mov Disord. 2006;21:325-331.

91. Benninger DH, Berman BD, Houdayer E, et al. Intermittent theta-burst transcranial magnetic stimulation for treatment of Parkinson disease. Neurology. 2011;76:601-609.

92. Spagnolo F, Volonté M, Fichera M, et al. Excitatory deep repetitive transcranial magnetic stimulation with $\mathrm{H}$-coil as add-on treatment of motor symptoms in Parkinson's disease: an open label, pilot study. Brain Stimul. 2014;7:297-300.

93. Cunnington R, Iansek R, Thickbroom GW, et al. Effects of magnetic stimulation over supplementary motor area on movement in Parkinson's disease. Brain. 1996;119:815-822.

94. Turner RS, Grafton ST, McIntosh AR, DeLong MR, Hoffman JM. The functional anatomy of parkinsonian bradykinesia. Neuroimage. 2003;19:163-179.

95. Brooks D, Piccini P, Turjanski N, Samuel M. Neuroimaging of dyskinesia. Ann Neurol. 2000;47:154-159.

96. Hoover JE, Strick PL. Multiple output channels in the basal ganglia. Science. 1993;259:819-821.

97. Cunnington R, Bradshaw JL, Iansek R. The role of the supplementary motor area in the control of voluntary movement. Hum Mov Sci. 1996;15:627-647.

98. Koch G, Brusa L, Caltagirone C, Peppe A. rTMS of supplementary motor area modulates therapy-induced dyskinesias in Parkinson disease. Neurology. 2005;65:623-625.

99. Brusa L, Versace V, Koch G, et al. Low frequency rTMS of the SMA transiently ameliorates peak-dose LID in Parkinson's disease. Clin Neurophysiol. 2006;117:1917-1921.

100. Sayın S, Cakmur R, Yener GG, Yaka E, Uğurel B, Uzunel F. Low-frequency repetitive transcranial magnetic stimulation for dyskinesia and motor performance in Parkinson's disease. J Clin Neurosci. 2014;21:1373-1376.

101. Shirota Y, Ohtsu H, Hamada M, Enomoto H, Ugawa Y. Supplementary motor area stimulation for Parkinson's disease: a randomized controlled study. Neurology. 2013;80:1400-1405.

102. Rizzo V, Siebner HR, Modugno N, et al. Shaping the excitability of human motor cortex with premotor rTMS. $J$ Physiol. 2003;554: 483-495.

103. Bäumer T, Lange R, Liepert J, et al. Repeated premotor rTMS leads to cumulative plastic changes of motor cortex excitability in humans. Neuroimage. 2003;20:550-560.

104. Munchau A, Bloem BR, Irlbacher K, Trimble MR, Rothwell JC. Functional connectivity of human premotor and motor cortex explored with repetitive transcranial magnetic stimulation. JNeurosci. 2002;22: 554-561.

105. Bäumer T, Hidding U, Hamel W, et al. Effects of DBS, premotor rTMS, and levodopa on motor function and silent period in advanced Parkinson's disease. Mov Disord. 2009;24:672-676.

106. Mir P, Matsunaga K, Quinn N, Siebner H, Rothwell J. Dopaminergic drugs restore facilitatory premotor-motor interactions in Parkinson's disease. Neurology. 2005;64:1906-1912.

107. Koch G, Brusa L, Carrillo F, et al. Cerebellar magnetic stimulation decreases levodopa-induced dyskinesias in Parkinson disease. Neurology. 2009;73:113-119. 
108. Minks E, Mareček R, Pavlík T, Ovesná P, Bareš M. Is the cerebellum a potential target for stimulation in Parkinson's disease? Results of 1-Hz rTMS on upper limb motor tasks. Cerebellum. 2011;10: 804-811.

109. Yu H, Sternad D, Corcos DM, Vaillancourt DE. Role of hyperactive cerebellum and motor cortex in Parkinson's disease. Neuroimage. 2007;35:222-233.

110. Lewis MM, Slagle CG, Smith AB, et al. Task specific influences of Parkinson's disease on the striato-thalamo-cortical and cerebello-thalamocortical motor circuitries. Neuroscience. 2007;147:224-235.

111. Ballanger B, Baraduc P, Broussolle E, Le Bars D, Desmurget M, Thobois S. Motor urgency is mediated by the contralateral cerebellum in Parkinson's disease. J Neurol Neurosurg Psychiatry. 2008;79: 1110-1116.

112. Hurley MJ, Mash DC, Jenner P. Markers for dopaminergic neurotrans mission in the cerebellum in normal individuals and patients with Parkinson's disease examined by RT-PCR. Eur J Neurosci. 2003;18: 2668-2672.

113. Vonsattel J, DiFiglia M. Huntington disease. J Neuropathol Exp Neurol. 1998;57:369-384.

114. [No authors lised]. A novel gene containing a trinucleotide repeat that is expanded and unstable on Huntingtons disease chromosomes. The Huntington's Disease Collaborative Research Group. Cell. 1993;72: 971-983.

115. Andrews TC, Weeks RA, Turjanski N, et al. Huntington's disease progression. PET and clinical observations. Brain. 1999;122 Pt 1: 2353-2363.

116. Douaud G, Behrens T, Poupon C, et al. In vivo evidence for the selective subcortical degeneration in Huntington's disease. Neuroimage. 2009;46:958-966.

117. Sapp E, Schwarz C, Chase K, et al. Huntingtin localization in brains of normal and Huntington's disease patients. Ann Neurol. 1997;42: 604-612.

118. Aron AR, Schlaghecken F, Fletcher PC, et al. Inhibition of subliminally primed responses is mediated by the caudate and thalamus: evidence from functional MRI and Huntington's disease. Brain. 2003;126: 713-723.

119. Meyer BU, Noth J, Lange HW, et al. Motor responses evoked by magnetic brain stimulation in Huntington's disease. Electroencephalogr Clin Neurophysiol. 1992;85:197-208.

120. Priori A, Berardelli A, Inghilleri M, Polidori L, Manfredi M. Electromyographic silent period after transcranial brain stimulation in Huntington's disease. Mov Disord. 1994;9:178-182.

121. Lefaucheur JP, Menard-Lefaucheur I, Maison P, et al. Electrophysiological deterioration over time in patients with Huntington's disease. Mov Disord. 2006;21:1350-1354.

122. Lefaucheur JP, Bachoud-Levi AC, Bourdet C, et al. Clinical relevance of electrophysiological tests in the assessment of patients with Huntington's disease. Mov Disord. 2002;17:1294-1301.

123. Schippling S, Schneider SA, Bhatia KP, et al. Abnormal motor cortex excitability in preclinical and very early Huntington's disease. Biol Psychiatry. 2009;65:959-965.

124. Brusa L, Versace V, Koch G, et al. Improvement of choreic movements by $1 \mathrm{~Hz}$ repetitive transcranial magnetic stimulation in Huntington's disease patients. Ann Neurol. 2005;58:655-656.

125. Shukla A, Jayarajan R, Muralidharan K, Jain S. Repetitive transcranial magnetic stimulation not beneficial in severe choreiform movements in Huntingon's disease. J ECT. 2013;29:e16-e17.

126. Vucic S, Ziemann U, Eisen A, Hallett M, Kiernan MC. Transcranial magnetic stimulation and amyotrophic lateral sclerosis: pathophysiological insights. J Neurol Neurosurg Psychiatry. 2013;84: 1161-1170.

127. Rowland L, Shneider N. Amyotrophic lateral sclerosis. N Engl J Med. 2001;344:1688-1700.

128. Vucic S, Cheah BC, Yiannikas C, Kiernan MC. Cortical excitability distinguishes ALS from mimic disorders. Clin Neurophysiol. 2011;122:1860-1866.
129. Schriefer T, Hess C, Mills K, Murray N. Central motor conduction studies in motor neurone disease using magnetic brain stimulation. Electroencephalogr Clin Neurophysiol. 1989;74:431-437.

130. Attarian S, Azulay J-P, Lardillier D, Verschueren A, Pouget J. Transcranial magnetic stimulation in lower motor neuron diseases. Clin Neurophysiol. 2005;116:35-42.

131. De Carvalho M, Turkman A, Swash M. Motor responses evoked by transcranial magnetic stimulation and peripheral nerve stimulation in the ulnar innervation in amyotrophic lateral sclerosis: the effect of upper and lower motor neuron lesion. J Neurol Sci. 2003;210(1/2): 83-90.

132. Triggs W, Menkes D, Onorato J, et al. Transcranial magnetic stimulation identifies upper motor neuron involvement in motor neuron disease. Neurology. 1999;53:605-611.

133. Mills KR, Nithi KA. Corticomotor threshold to magnetic stimulation: normal values and repeatability. Muscle Nerve. 1997;20: 570-576.

134. Zanette G, Tamburin S, Manganotti P, Refatti N, Forgione A, Rizzuto N. Different mechanisms contribute to motor cortex hyperexcitability in amyotrophic lateral sclerosis. Clin Neurophysiol. 2002;113:1688-1697.

135. Karandreas N, Papadopoulou M, Kokotis P, Papapostolou A, Tsivgoulis G, Zambelis T. Impaired interhemispheric inhibition in amyotrophic lateral sclerosis. Amyotroph Lateral Scler. 2007;8: $112-118$.

136. Zanette G, Tamburin S, Manganotti P, Refatti N, Forgione A, Rizzuto N Changes in motor cortex inhibition over time in patients with amyotrophic lateral sclerosis. J Neurol. 2002;249:1723-1728.

137. Wittstock M, Wolters A, Benecke R. Transcallosal inhibition in amyotrophic lateral sclerosis. Clin Neurophysiol. 2007;118:301-307.

138. Yokota T, Yoshino A, Inaba A, Saito Y. Double cortical stimulation in amyotrophic lateral sclerosis. J Neurol Neurosurg Psychiatry. 1996;61: 596-600.

139. Enterzari-Taher M, Eisen A, Stewart H, Nakajima M. Abnormalities of cortical inhibitory neurons in amyotrophic lateral sclerosis. Muscle Nerve. 1997;20:65-71.

140. De Carvalho M, Scotto M, Lopes A, Swash M. Clinical and neurophysiological evaluation of progression in amyotrophic lateral sclerosis. Muscle Nerve. 2003;28:630-633.

141. Floyd AG, Yu QP, Piboolnurak P, et al. Transcranial magnetic stimulation in ALS: utility of central motor conduction tests. Neurology. 2009; 72:498-504.

142. Angelucci F, Oliviero A, Pilato F. Transcranial magnetic stimulation and BDNF plasma levels in amyotrophic lateral sclerosis. Neuroreport. 2004; 15:717-720.

143. Di Lazzaro V, Oliviero A, Saturno E, et al. Motor cortex stimulation for amyotrophic lateral sclerosis. time for a therapeutic trial? Clin Neurophysiol. 2004;115:1479-1485.

144. Di Lazzaro V, Dileone M, Pilato F, et al. Repetitive transcranial magnetic stimulation for ALS. A preliminary controlled study. Neurosci Lett. 2006;408:135-140.

145. Zanette G, Forgione A, Manganotti P, Fiaschi A, Tamburin S. The effect of repetitive transcranial magnetic stimulation on motor performance, fatigue and quality of life in amyotrophic lateral sclerosis. $J$ Neurol Sci. 2008;270:18-22.

146. Di Lazzaro V, Pilato F, Profice P, et al. Motor cortex stimulation for ALS: a double blind placebo-controlled study. Neurosci Lett. 2009;464:18-21.

147. Dileone M, Profice P, Pilato F, et al. Repetitive transcranial magnetic stimulation for ALS. CNS Neurol Disord Drug Targets. 2010;9: 331-334.

148. Di Lazzaro V, Dileone M, Pilato F, et al. Long-term motor cortex stimulation for amyotrophic lateral sclerosis. Brain Stimul. 2010;3: 22-27.

149. Fang J, Zhou M, Yang M, Zhu C, He L. Repetitive transcranial magnetic stimulation for the treatment of amyotrophic lateral sclerosis or motor neuron disease. Cochrane Database Syst Rev. 2013;5:CD008554. 
150. Eisen A, Shytbel W, Murphy K, Horich M. Cortical magnetic stimulation in amyotrophic lateral sclerosis. Muscle Nerve. 1990;13: 146-151.

151. Claus D, Brunhölzl C, Kerling FP, Henschel S. Transcranial magnetic stimulation as a diagnostic and prognostic test in amyotrophic lateral sclerosis. J Neurol Sci. 1995;Suppl 129:30-34.

152. Geevasinga N, Menon P, Yiannikas C, Kiernan MC, Vucic S. Diagnostic utility of cortical excitability studies in amyotrophic lateral sclerosis. Eur J Neurol. April 2, 2014. [Epub ahead of print.]

153. Miscio G, Pisano F, Mora G, Mazzini L. Motor neuron disease: usefulness of transcranial magnetic stimulation in improving the diagnosis. Clin Neurophysiol. 1999;110:975-981.

154. Schulte-Mattler WJ, Müller T, Zierz S. Transcranial magnetic stimulation compared with upper motor neuron signs in patients with amyotrophic lateral sclerosis. J Neurol Sci. 1999; 170:51-56

155. Pohl C, Block W, Träber F, et al. Proton magnetic resonance spectroscopy and transcranial magnetic stimulation for the detection of upper motor neuron degeneration in ALS patients. J Neurol Sci. 2001;190:21-27.

156. Vucic S, Lin CS-Y, Cheah BC, et al. Riluzole exerts central and peripheral modulating effects in amyotrophic lateral sclerosis. Brain. 2013;136 Pt 5:1361-1370.

157. Eisen A, Pant B, Stewart H. Cortical excitability in amyotropic lateral sclerosis: a clue to pathogenesis. Can J Neurol Sci. 1993;20:11-16.

158. Stefan K, Kunesch E, Benecke R, Classen J. Effects of riluzole on cortical excitability in patients with amyotrophic lateral sclerosis. Ann Neurol. 2001;49:536-539.

159. Vucic S, Kiernan MC. Novel threshold tracking techniques suggest that cortical hyperexcitability is an early feature of motor neuron disease. Brain. 2006;129 Pt 9:2436-2446.

160. Vucic S, Kiernan MC. Upregulation of persistent sodium conductances in familial ALS. J Neurol Neurosurg Psychiatry. 2010;81:222-227.

161. Khedr EM, Ahmed MA, Hamdy A, Shawky OA. Cortical excitability of amyotrophic lateral sclerosis: transcranial magnetic stimulation study. Neurophysiol Clin. 2011;41:73-79.

162. Mills KR. The natural history of central motor abnormalities in amyotrophic lateral sclerosis. Brain. 2003;126 Pt 11:2558-2566.

163. Vucic S, Cheah BC, Kiernan MC. Maladaptation of cortical circuits underlies fatigue and weakness in ALS. Amyotroph Lateral Scler. 2011;12:414-420.

164. De Carvalho M, Scotto M, Lopes A, Swash M. The corticomotor threshold is not dependent on disease duration in amyotrophic lateral sclerosis (ALS). Amyotroph Lateral Scler Other Mot Neuron Disord. 2002;3:39-42.

165. Urban PP, Wicht S, Hopf HC. Sensitivity of transcranial magnetic stimulation of cortico-bulbar vs cortico-spinal tract involvement in amyotrophic lateral sclerosis (ALS). J Neurol. 2001;248:850-855.

166. Desiato MT, Bernardi G, Hagi HA, Boffa L, Caramia MD. Transcranial magnetic stimulation of motor pathways directed to muscles supplied by cranial nerves in amyotrophic lateral sclerosis. Clin Neurophysiol. 2002;113:132-140.

167. Urban P, Vogt T, Hopf H. Corticobulbar tract involvement in amyotrophic lateral sclerosis. A transcranial magnetic stimulation study. Brain. 1998;121 Pt 6:1099-1108.

168. Magistris MR, Rösler KM, Truffert A, Myers JP. Transcranial stimulation excites virtually all motor neurons supplying the target muscle. A demonstration and a method improving the study of motor evoked potentials. Brain. 1998;121 Pt 3:437-450.

169. Komissarow L. Triple stimulation technique (TST) in amyotrophic lateral sclerosis. Clin Neurophysiol. 2004;115:356-360.

170. Rösler K, Truffert A, Hess C, Magistris M. Quantification of upper motor neuron loss in amyotrophic lateral sclerosis. Clin Neurophysiol. 2000;111:2208-2218.

171. Bühler R, Magistris MR, Truffert A, Hess CW, Rösler KM. The triple stimulation technique to study central motor conduction to the lower limbs. Clin Neurophysiol. 2001;112:938-949.
172. Kale N, Agaoglu J, Onder G, Tanik O. Correlation between disability and transcranial magnetic stimulation abnormalities in patients with multiple sclerosis. J Clin Neurosci. 2009;16:1439-1442.

173. Mori F, Kusayanagi H, Monteleone F, et al. Short interval intracortical facilitation correlates with the degree of disability in multiple sclerosis. Brain Stimul. 2013;6:67-71.

174. Tataroglu C, Genc A, Idiman E, Cakmur R, Idiman F. Cortical silent period and motor evoked potentials in patients with multiple sclerosis. Clin Neurol Neurosurg. 2003;105:105-110.

175. McDonald WI, Compston A, Edan G, et al. Recommended diagnostic criteria for multiple sclerosis: guidelines from the International Panel on the diagnosis of multiple sclerosis. Ann Neurol. 2001;50: 121-127.

176. Lassmann H, Brück W, Lucchinetti CF. The immunopathology of multiple sclerosis: an overview. Brain Pathol. 2007;17:210-218.

177. Weinshenker BG, Bass B, Rice GP, et al. The natural history of multiple sclerosis: a geographically based study. I. Clinical course and disability. Brain. 1989;112:133-146.

178. Filippi M, Campi A, Mammi S, et al. Brain magnetic resonance imaging and multimodal evoked potentials in benign and secondary progressive multiple sclerosis. J Neurol Neurosurg Psychiatry. 1995;58: 31-37.

179. Hume AL, Waxman SG. Evoked potentials in suspected multiple sclerosis: diagnostic value and prediction of clinical course. J Neurol Sci. 1988;83:191-210.

180. Schmierer K, Irlbacher K, Grosse P, Röricht S, Meyer B. Correlates of disability in multiple sclerosis detected by transcranial. Neurology. 2002;59:1218-1224.

181. Caramia MD, Palmieri MG, Desiato MT, et al. Brain excitability changes in the relapsing and remitting phases of multiple sclerosis: a study with transcranial magnetic stimulation. Clin Neurophysiol. 2004;115:956-965.

182. Fuhr P, Kappos L. Evoked potentials for evaluation of multiple sclerosis. Clin Neurophysiol. 2001;112:2185-2189.

183. Mori F, Kusayanagi H, Nicoletti CG, Weiss S, Marciani MG, Centonze D. Cortical plasticity predicts recovery from relapse in multiple sclerosis. Mult Scler. 2014;20:451-457.

184. Rizzo M, Hadjimichael O, Preiningerova J, Vollmer T. Prevalence and treatment of spasticity reported by multiple sclerosis patients. Mult Scler. 2004;10:589-595.

185. Nielsen J, Sinkjaer T, Jakobsen J. Treatment of spasticity with repetitive magnetic stimulation; a double-blind placebo-controlled study. Mult Scler. 1996;2:227-232.

186. Chen R, Garg R, Lozano A, Lang A. Effects of internal globus pallidus stimulation on motor cortex excitability. Neurology. 2001;56: 716-723.

187. Pierantozzi M, Palmieri M, Marciani M, Bernardi G, Giacomini P, Stanzione P. Effect of apomorphine on cortical inhibition in Parkinson's disease patients: a transcranial magnetic stimulation study. Exp Brain Res. 2001;141:52-62.

188. Li J-Y, Espay AJ, Gunraj CA, et al. Interhemispheric and ipsilateral connections in Parkinson's disease: relation to mirror movements. $\mathrm{Mov}$ Disord. 2007;22:813-821.

189. Abbruzzese G, Buccolieri A, Marchese R, Trompetto C, Mandich P, Schieppati M. Intracortical inhibition and facilitation are abnormal in Huntington's disease: a paired magnetic stimulation study. Neurosci Lett. 1997;228:87-90.

190. Hömberg V, Lange HW. Central motor conduction to hand and leg muscles in Huntington's disease. Mov Disord. 1990;5: 214-218.

191. Ziemann U, Winter M, Reimers C, Riemers K, Tergau F, Paulus W. Impaired motor cortex inhibition in patients with amyotrophic lateral sclerosis. Evidence from paried transcranial magnetic stimulation. Neurology. 1997;49:1292-1298.

192. Hanajima R, Ugawa Y, Terao Y, Ogata K, Kanazawa I. Ipsilateral cortico-cortical inhibition of the motor cortex in various neurological disorders. J Neurosci. 1996;140:109-116. 
193. Vucic S, Cheah BC, Kiernan MC. Defining the mechanisms that underlie cortical hyperexcitability in amyotrophic lateral sclerosis. Exp Neurol. 2009;220:177-182.

194. Lenzi D, Conte A, Mainero C, et al. Effect of corpus callosum damage on ipsilateral motor activation in patients with multiple sclerosis: a functional and anatomical study. Hum Brain Mapp. 2007;28: 636-644.

195. Petajan JH, White AT. Motor-evoked potentials in response to fatiguing grip exercise in multiple sclerosis patients. Clin Neurophysiol. 2000;111:2188-2195.

196. Filipović SR, Rothwell JC, Bhatia K. Slow $(1 \mathrm{~Hz})$ repetitive transcranial magnetic stimulation (rTMS) induces a sustained change in cortical excitability in patients with Parkinson's disease. Clin Neurophysiol. 2010;121:1129-1137.

197. Gilio F, Currà A, Inghilleri M, Lorenzano C, Manfredi M, Berardelli A. Repetitive magnetic stimulation of cortical motor areas in Parkinson's disease: implications for the pathophysiology of cortical function. Mov Disord. 2002;17:467-473.

198. Benninger DH, Iseki K, Kranick S, Luckenbaugh DA, Houdayer E, Hallett M. Controlled study of 50-Hz repetitive transcranial magnetic stimulation for the treatment of Parkinson disease. Neurorehabil Neural Repair. 2012;26:1096-1105.

199. Eggers C, Fink GR, Nowak DA. Theta burst stimulation over the primary motor cortex does not induce cortical plasticity in Parkinson's disease. J Neurol. 2010;257:1669-1674.

200. Suppa A, Marsili L, Belvisi D, et al. Lack of LTP-like plasticity in primary motor cortex in Parkinson's disease. Exp Neurol. 2011;227: 296-301.

201. Morgante F, Espay AJ, Gunraj C, Lang AE, Chen R. Motor cortex plasticity in Parkinson's disease and levodopa-induced dyskinesias. Brain. 2006;129 Pt 4:1059-1069.

202. Ueki Y, Mima T, Kotb MA, et al. Altered plasticity of the human motor cortex in Parkinson's disease. Ann Neurol. 2006;59:60-71.

203. Kačar A, Filipović SR, Kresojević N, et al. History of exposure to dopaminergic medication does not affect motor cortex plasticity and excitability in Parkinson's disease. Clin Neurophysiol. 2013;124: 697-707.

204. Bagnato S, Agostino R, Modugno N, Quartarone A, Berardelli A. Plasticity of the motor cortex in Parkinson's disease patients on and off therapy. Mov Disord. 2006;21:639-645.

205. Orth M, Schippling S, Schneider SA, et al. Abnormal motor cortex plasticity in premanifest and very early manifest Huntington disease. J Neurol Neurosurg Psychiatry. 2010;81:267-270.

206. Crupi D, Ghilardi MF, Mosiello C, Di Rocco A, Quartarone A, Battaglia F. Cortical and brainstem LTP-like plasticity in Huntington's disease. Brain Res Bull. 2008;75:107-114.

207. Mori F, Codecà C, Kusayanagi H, et al. Effects of intermittent theta burst stimulation on spasticity in patients with multiple sclerosis. Eur J Neurol. 2010;17:295-300.

208. Centonze D, Koch G, Versace V, et al. Repetitive transcranial magnetic stimulation of the motor cortex ameliorates spasticity in multiple sclerosis. Neurology. 2007;68:1045-1050.

209. Mori F, Ljoka C, Magni E, et al. Transcranial magnetic stimulation primes the effects of exercise therapy in multiple sclerosis. J Neurol. 2011;258:1281-1287.
210. Koch G, Rossi S, Prosperetti C, et al. Improvement of hand dexterity following motor cortex rTMS in multiple sclerosis patients with cerebellar impairment. Mult Scler. 2008;14:995-998.

211. Priori A, Polidori L, Rona S, Manfredi M, Berardelli A. Spinal and cortical inhibition in Huntington's chorea. Mov Disord. 2000;15: 938-946.

212. Modugno N, Currà A, Giovannelli M, et al. The prolonged cortical silent period in patients with Huntington's disease. Clin Neurophysiol. 2001;112:1470-1474.

213. Lorenzano C, Dinapoli L, Gilio F, et al. Motor cortical excitability studied with repetitive transcranial magnetic stimulation in patients with Huntington's disease. Clin Neurophysiol. 2006;117:1677-1681.

214. Tegenthoff M, Vorgerd M, Juskowiak F, Roos V, Malin JP. Postexcitatory inhibition after transcranial magnetic single and double brain stimulation in Huntington's disease. Electroencephalogr Clin Neurophysiol. 1996;101:298-303.

215. Eisen A, Bolega S, Bloch M, Hayden M. Silent periods, longlatency reflexes, and cortical MEPs in Huntington's disease and at-risk relatives. Electroencephalogr Clin Neurophysiol. 1989;74: 444-449.

216. Triggs W, Macdonell R, Cros D, Chiappa K, Shahani B, Day B. Motor inhibition and excitation are independent effects of magnetic cortical sitmulation. Ann Neurol. 1992;32:341-351.

217. Berardelli A, Inghilleri M, Cruccu G, Mercuri B, Manfredi M. Electrical and magneticc transcranial stimulation in patients with corticospinal damage due to stroke or motor neurone disease. Electroencephalogr Clin Neurophysiol. 1991;81:389-396.

218. Naka D, Mills KR. Further evidence for corticomotor hyperexcitability in amyotrophic lateral sclerosis. Muscle Nerve. 2000;23:1044-1050.

219. Caramia MD, Cicinelli P, Paradiso C, et al. Excitability changes of muscular responses to magnetic brain stimulation in patients with central motor disorders. Electroencephalogr Clin Neurophysiol. 1991;81:243-250.

220. Kohara N, Kaji R, Kojima Y, et al. Abnormal excitability of the corticospinal pathway in patients with amyotrophic lateral sclerosis: a single motor unit study using transcranial magnetic stimulation. Electroencephalogr Clin Neurophysiol. 1996;101:32-41.

221. De Carvalho M, Miranda PC, Luís ML, Ducla-Soares E. Cortical muscle representation in amyotrophic lateral sclerosis patients: changes with disease evolution. Muscle Nerve. 1999;22:1684-1692.

222. Prout A, Eisen A. The cortical silent period and amyotrophic lateral sclerosis. Muscle Nerve. 1994;17:217-223.

223. Uozumi T, Tsuji S, Murai Y. Motor potentials evoked by magnetic stimulation of the motor cortex in normal subjects and patients with motor disorders. Electroencephalogr Clin Neurophysiol. 1991;81:251-256.

224. Vucic S, Kiernan MC. Cortical excitability testing distinguishes Kennedy's disease from amyotrophic lateral sclerosis. Clin Neurophysiol. 2008;119:1088-1096.

225. Siciliano G, Manca ML, Sagliocco L, et al. Cortical silent period in patients with amyotrophic lateral sclerosis. J Neurol Sci. 1999;169:93-97.

226. Wittstock M, Meister S, Walter U, Benecke R, Wolters A. Mirror movements in amyotrophic lateral sclerosis. Amyotroph Lateral Scler. 2011;12:393-397.
Degenerative Neurological and Neuromuscular Disease

\section{Publish your work in this journal}

Degenerative Neurological and Neuromuscular Disease is an international, peer-reviewed, open access journal focusing on research into degenerative neurological and neuromuscular disease, identification of therapeutic targets and the optimal use of preventative and integrated treatment interventions to achieve improved outcomes,

\section{Dovepress}

enhanced survival and quality of life for the patient. The manuscript management system is completely online and includes a very quick and fair peer-review system. Visit http://www.dovepress.com/testimonials.php to read real quotes from published authors. 